\title{
WELLPOSEDNESS OF A NONLINEAR, LOGARITHMIC SCHRÖDINGER EQUATION OF DOEBNER-GOLDIN TYPE MODELING QUANTUM DISSIPATION
}

\author{
P. GUERRERO, J. L. LÓPEZ, J. MONTEJO-GÁMEZ, AND J. NIETO
}

\begin{abstract}
This paper is concerned with the modeling and analysis of quantum dissipation phenomena in the Schrödinger picture. More precisely, we do investigate in detail a dissipative, nonlinear Schrödinger equation somehow accounting for quantum Fokker-Planck effects, and how it is drastically reduced to a simpler logarithmic equation via a nonlinear gauge transformation in such a way that the physics underlying both problems keeps unaltered. From a mathematical viewpoint, this allows for a more achievable analysis regarding the local wellposedness of the initial-boundary value problem. This simplification requires the performance of the polar (modulus-argument) decomposition of the wavefunction, which is rigorously attained (for the first time to the best of our knowledge) under quite reasonable assumptions.
\end{abstract}

\section{Introduction, Setting of the PRoblem And main RESUlt}

Quantum dissipation and diffusion modeling has been widely analyzed over last years, mainly in the context of open quantum systems. We refer for instance to $[6,7,9,22,31,41]$. Recently, the quantum-mechanical treatment of dissipative processes and other nonequilibrium phenomena has been a subject of much attention due to its applicability in various fields such as solid state and statistical physics, photochemistry, Brownian dynamics, heavy ion scattering, quantum gravity theories or ecology. In the Schrödinger picture, quantum friction and diffusion effects have been more or less succeedingly modeled by nonlinear terms of type $\lambda S_{\psi} \psi$ (formulated by Kostin [31] to describe nonlinear Schrödinger-Langevin dynamics), where $\lambda$ is a friction constant and $S_{\psi}=-i \log (\psi /|\psi|)$ stands for the (multivalued) argument of the complex wavefunction $\psi(t, x)$, as well as by logarithmic nonlinearities with the form $\log \left(|\psi|^{2}\right) \psi$ (first studied by Bialinicki-Birula and Mycielski in [9]), among others (see for example [6, 13, 15, 27, 34]). A large number of the nonlinear Schrödinger equations proposed in the literature involve complex nonlinearities describing different phenomenologies in condensed matter physics, e.g. incoherent solitons, dynamical modes of plasma physics, propagation of optical pulses or damping effects in nonlinear media. For instance, the

Key words and phrases. Wigner-Fokker-Planck equation; Doebner-Goldin equations; dissipative quantum mechanics; nonlinear Schrödinger equation; logarithmic nonlinearities; local solvability; Madelung transformation; reconstruction of the wavefunction. 
Doebner-Goldin equations

$$
\begin{aligned}
i \hbar \partial_{t} \psi= & -\frac{\hbar^{2}}{2 m} \Delta_{x} \psi+\frac{i \hbar D}{2}\left(\frac{\Delta_{x} n}{n}\right) \psi+V \psi+m D^{\prime} \mu_{1}\left(\frac{\nabla_{x} \cdot J}{n}\right) \psi \\
& +\frac{m^{2}}{\hbar} D^{\prime} \mu_{3}\left(\frac{|J|}{n}\right)^{2} \psi+m D^{\prime} \mu_{4}\left(\frac{J \cdot \nabla_{x} n}{n^{2}}\right) \psi \\
& +\hbar D^{\prime}\left(\mu_{2} \frac{\Delta_{x} n}{n}+\mu_{5} \frac{\left|\nabla_{x} n\right|^{2}}{n^{2}}\right) \psi
\end{aligned}
$$

were introduced in [22] as the most general class of Schrödinger type equations compatible with the Fokker-Planck continuity equation for the probability density $n=|\psi|^{2}$, namely $\partial_{t} n+\nabla_{x} \cdot J=D \Delta_{x} n$, with $J(t, x)$ denoting the electric current. The subfamily of these equations characterized by the identities

$$
D^{\prime} \mu_{1}=D=-D^{\prime} \mu_{4}, \quad \mu_{2}+2 \mu_{5}=0, \quad \mu_{3}=0,
$$

satisfies the Ehrenfest theorem of quantum mechanics and was shown to be linearizable through a suitable nonlinear transformation (see $[6,23,36])$ if the constraint

$$
\frac{4 m D^{\prime}}{\hbar} \mu_{2}<1-\frac{4 m^{2} D^{2}}{\hbar^{2}}
$$

is fulfilled. The nonlinearities of the Doebner-Goldin equations were derived in the frame of group theory, more precisely from the representation analysis of the quantum kinematical group of diffeomorphisms $\operatorname{Diff}\left(\mathbb{R}^{3}\right)$. Very recently a nonlinear logarithmic Schrödinger equation of Doebner-Goldin type was introduced and analyzed by two of us in [35] from a hydrodynamic perspective, starting from the Wigner-Fokker-Planck system (see e.g. $[4,5,11]$ for a physical motivation and some mathematical analysis concerning the wellposedness of the latter model coupled to Poisson's equation).

In this paper we investigate the following nonlinear Schrödinger equation of logarithmic type (that might be called 'Full Logarithmic Schrödinger Equation' or, in short, FLSE for future reference), derived in [34] in the one-dimensional case and in full generality in [28] under a Nelsonian stochastic approach, which rules the evolution in $(t, x) \in[0, T) \times \Omega$ of a quantum electron gas interacting with a heat bath in thermodynamic equilibrium

$$
\begin{aligned}
i \alpha \partial_{t} \psi & =\left(-\frac{\alpha^{2}}{2 m} \Delta_{x}+V_{\alpha}\right) \psi, \\
V_{\alpha} & =\frac{\alpha^{2}}{\hbar^{2}} Q+\Lambda \log (n)+\frac{\alpha}{2 m}\left(\frac{i \alpha}{2} \frac{\Delta_{x} n}{n}+m \nabla_{x} \cdot \frac{J}{n}\right),
\end{aligned}
$$

with initial and boundary conditions given by

$$
\begin{aligned}
& \psi(0, x)=\psi_{0}(x), \quad x \in \Omega, \\
& \psi(t, x)=\psi_{B}(x), \quad x \in \partial \Omega, \quad t \in[0, T),
\end{aligned}
$$


where $\psi=\psi(t, x)$ is the (complex) wavefunction,

$$
\alpha=2 m D_{q q}, \quad \Lambda=2 D_{p q}+\eta D_{q q},
$$

$\Omega$ is a smooth bounded domain in $\mathbb{R}^{3}$ (though all of our results can be also stated without changes in the case $\left.\Omega \subset \mathbb{R}^{d}, 1 \leq d \leq 3\right), m$ is the effective mass of the electrons and where

$$
D_{p q}=\frac{\eta \omega \hbar^{2}}{12 \pi m k_{B} T}, \quad D_{q q}=\frac{\eta \hbar^{2}}{12 m^{2} k_{B} T}
$$

are phenomenological (diffusion) constants related to the system-reservoir interactions. Here, $\hbar$ is the reduced Planck constant, $\eta=2 m \lambda$ is the damping/coupling constant of the thermal bath, $\lambda$ is the friction coefficient, $\omega$ is the cut-off frequency of the reservoir oscillators, $k_{B}$ is the Boltzmann constant and $T$ is the bath temperature. Also, $Q(t, x)$ denotes Bohm's quantum potential defined by

$$
Q:=-\frac{\hbar^{2}}{2 m}\left(\frac{\Delta_{x} \sqrt{n}}{\sqrt{n}}\right)=-\frac{\hbar^{2}}{4 m}\left(\frac{\Delta_{x} n}{n}-\frac{\left|\nabla_{x} n\right|^{2}}{2 n^{2}}\right),
$$

$n=|\psi|^{2}$ holds for the local density and

$$
J=\frac{\alpha}{m} \operatorname{Im}\left(\bar{\psi} \nabla_{x} \psi\right)
$$

is the electric current, $\operatorname{Im}(z)$ and $\bar{z}$ denoting imaginary part and complex conjugation respectively. The electron Hamiltonian $H_{\alpha}$ may appear augmented by an external potential $V=V(t, x)$, typically the quantum harmonic oscillator confining potential $V=\frac{m}{2} \omega_{0}^{2}|x|^{2}$, with $\omega_{0}$ standing for the oscillator frequency, although nonlinear and self-consistent couplings can be also considered.

The quantum correction involving Bohm's potential in Eq. (4), which gives rise to a modular type nonlinearity $\kappa Q \psi[6,25]$, represents a field through which the electrons interact with themselves and can be interpreted as a quantum diffusion term yielding a theory which contains quantum-mechanical confinement effects. This potential has been used, for example, to study wave packet tunnelling through barriers. On the other hand, meaningful physical interpretations have been also given to the presence of the logarithmic potential $\log (n)$ in the Schrödinger equation. Indeed, it can be understood as the effect of statistical uncertainty or as the potential energy associated with the information encoded in the matter distribution described by the probability density $n(t, x)$. The logarithmic nonlinearity has been recently proposed for the modeling of several phenomena occurring in capillary fluids and magma transport [20, 21, 32]. Furthermore, Eq. (4) retains a nonlinear complex potential describing quantum position diffusion. This is in good agreement with the physical interpretation of complex potentials, as they have been used in the literature to simulate dissipative processes and decoherence effects in the transition regions of small quantum devices. 
The Schrödinger system (3)-(4) can be viewed as a particular subclass of the Doebner-Goldin equations (with action unit $\alpha$ instead of $\hbar$ ) belonging to the Ehrenfest family (cf. (1)) and containing an additional logarithmic nonlinearity, by just identifying

$$
\begin{aligned}
& D=D_{q q}, \quad \mu_{3}=0, \quad D^{\prime} \mu_{1}=D_{q q}=-D^{\prime} \mu_{4}, \\
& D^{\prime} \mu_{2}=-\frac{D_{q q}}{2}=-2 D^{\prime} \mu_{5} .
\end{aligned}
$$

Besides, the linearization condition (2) is also fulfilled (with $\alpha$ instead of $\hbar$ ). As a matter of fact, the nonlinearities in (4) can be drastically simplified to just the logarithmic one still preserving the underlying physics. In particular, if considering the Madelung form of the wavefunction

$$
\psi(t, x)=\sqrt{n_{\psi}(t, x)} \exp \left\{\frac{i}{\alpha} S_{\psi}(t, x)\right\},
$$

then the nonlinear change of phase $\psi \mapsto \phi=F(\psi)$ defined by

$$
\begin{aligned}
F(\psi)(t, x) & =\sqrt{n_{\psi}(t, x)} \exp \left\{-i \log \left(\sqrt{n_{\psi}(t, x)}\right)+\frac{1}{\alpha} S_{\psi}(t, x)\right\} \\
& =\psi(t, x) \exp \left\{-\frac{i}{2} \log \left(n_{\psi}(t, x)\right)\right\}
\end{aligned}
$$

is a (formal by the moment) one-to-one correspondence between solutions of the FLSE and solutions of the following 'purely logarithmic Schrödinger equation' (PLSE)

$$
i \alpha \partial_{t} \phi=-\frac{\alpha^{2}}{2 m} \Delta_{x} \phi+\Lambda \log (n) \phi .
$$

The transformation $F$ defined in (11) does belong to a general class of mappings known as nonlinear gauge transforms, which preserve some fundamental aspects of quantum mechanics such as the probability density $n(t, x)$. We are intended to reduce (via $F$ ) the wellposedness problem associated with Eq. (3)-(6) to the study of Eq. (12) with the following boundary and initial conditions

$$
\begin{aligned}
& \phi(0, x)=\phi_{0}(x), \quad x \in \Omega, \\
& \phi(t, x)=\phi_{B}(x), \quad x \in \partial \Omega, \quad t \in[0, T) .
\end{aligned}
$$

Similar strategies were also recently developed in [16, 38], in the scope of derivative Schrödinger equations.

Some wellposedness and stability properties were shown in $[12,13,14]$ for a class of logarithmic Schrödinger equations, where the logarithmic nonlinearity was considered with opposite sign to that explored in this paper. Nevertheless, this fact drastically changes the dynamics of the system (see [28, 34], where a comparison between the phase portraits of both stationary systems was performed). For instance, in that situation one finds soliton-like solutions with 
Gaussian shape (Gaussons) in any number of dimensions, describing the propagation of nonspreading wave packets of freely moving particles [9]. In fact, the radial Gausson was shown in [13] to be orbitally stable under radial perturbations. The single sign choice for the logarithmic term first made in [9] and later continued in $[13,14]$ was owing to the fact that the other sign led to an energy functional which was not bounded from below. However, the positive sign for the logarithmic nonlinearity was also physically justified in [18] as representing a diffusion force within the context of stochastic quantum mechanics [8,37], and mathematically analyzed in the whole space in [29]. Besides, a simple minimization argument for slow varying, compactly supported density profiles was carried out by Davidson in [19] to conclude that the (usual) assumption about the sign of the logarithmic term made in [9] is not the only reasonable possibility and that a sensible theory can be developed with the opposite sign as well, as argued herein.

Our strategy will consist in developing a fixed-point argument on an appropriate subset of $H^{2}(\Omega)$, consisting of those wavefunctions living far from vacuum, in order to obtain regular solutions to Eq. (12)-(14), thus to Eq. (3)-(6) by means of $F$ (cf. formula (11)). This analytical treatment does require an accurate definition of $S_{\psi}$ due to the multivaluedness of the complex logarithm. The required assumptions for our analysis are

(H1) $\Omega \subset \mathbb{R}^{3}$ is a simply-connected, $C^{2}$ bounded domain.

(H2) $\psi_{0} \in H^{2}(\Omega), \psi_{B} \in H^{3 / 2}(\partial \Omega)$, and $\psi_{0}=\psi_{B}$ in $\partial \Omega$.

(H3) There exists $\delta>0$ such that

$$
\operatorname{ess-inf}\left\{\left|\psi_{0}(x)\right|: x \in \Omega\right\}>\delta, \quad \operatorname{ess-inf}\left\{\left|\psi_{B}(x)\right|: x \in \partial \Omega\right\}>\delta .
$$

Note that the Rellich-Kondrachov compactness theorem (see for example [10]) along with the regularity properties of $\psi_{0}$ and $\psi_{B}$, allow us to consider the condition $\psi_{0}=\psi_{B}$ stated in (H2) in the usual sense, $\psi_{0}-\psi_{B} \in H_{0}^{1}(\Omega)$, as well as a pointwise identity in $\partial \Omega$.

We start by fixing some notation that will be useful in the sequel. Let $H$ be a subset of $L^{2}(\Omega), \delta$ a positive constant, and denote

$$
H_{\delta}=\{\varphi \in H:|\varphi|>\delta \text { a.e. in } \Omega\} .
$$

Given $T>0$, we also denote

$$
X^{T}=C\left([0, T) ; H^{2}(\Omega)\right) \cap C^{1}\left([0, T) ; L^{2}(\Omega)\right)
$$

and

$$
X_{\delta}^{T}=\left\{\varphi \in X^{T}:|\varphi|>\delta \text { a.e. } x \in \Omega, \forall 0 \leq t<T\right\},
$$

where $H^{k}(\Omega)$ is the usual Sobolev space $W^{k, 2}(\Omega)$.

Finally, given $\varphi \in H^{1}(\Omega)$ we write $n_{\varphi}=|\varphi|^{2}$ and $J_{\varphi}=\frac{\alpha}{m} \operatorname{Im}\left(\bar{\varphi} \nabla_{x} \varphi\right)$ (or simply $n$ and $J$ when an unique wavefunction is involved). In the following, 
we shall simply denote $\nabla$ and $\Delta$ the gradient and Laplace operators when no possible confusion concerning the variable with respect to which differentiation is being performed can arise. Otherwise we will denote $\nabla_{x}$ and $\Delta_{x}$ to indicate differentiation with respect to the position coordinate. Our main result reads as follows.

Theorem 1.1. Assume that (H1)-(H3) hold. Then, the following properties are satisfied

(i) There exists $T=T\left(\delta, \psi_{0}, \psi_{B}, \Omega\right)>0$ such that the nonlinear Schrödinger initial-boundary value problem (3)-(6) admits a unique strong solution $\psi \in X_{\delta}^{T}$

(ii) The dynamics underlying (3)-(6) is (topologically) equivalent to that associated with the initial-boundary value problem consisting of Eq. (12) subject to the conditions (13)-(14), in the sense that there exists a suitable homeomorphism in $C\left([0, T) ; H_{\delta}^{2}\right)$ that carries strong solutions of $(3)-(6)$ into strong solutions of (12)-(14).

The contents of the paper are structured as follows: In Section 2 we give an overview of the stochastic derivation of our main equation (see [28] for details). Section 3 is devoted to a detailed discussion and analysis of the main mathematical difficulties arising when dealing with Madelung's representation of the wavefunction, mainly consisting of the multivaluedness of the argument of the wavefunction. In particular the existence of regular arguments shall be proved, which is of fundamental importance for our research. In Section 4 we show how the FLSE is topologically equivalent to the standard PLSE through the action of the homeomorphic transformation. In Section 5 we address the local wellposedness issue for the mixed initial-boundary value (auxiliary) problem associated with the PLSE. Finally, in Section 6 the three-dimensional local solvability of the FLSE is proved on bounded domains satisfying (H1), starting from that for the PLSE, by carefully 'transporting' the wellposedness result shown for the latter along $F^{-1}$.

\section{On the multidimensional DeRivation of EQ. (3)}

In this section we review the main aspects of the three-dimensional derivation of Eq. (3)-(4) carried out in [28] (see also [34] for the 1D derivation). The starting point is the Wigner-Fokker-Planck equation

$$
\partial_{t} w+\left(\xi \cdot \nabla_{x}\right) w+\theta_{V}[w]=L_{Q F P}[w],
$$

with

$$
L_{Q F P}[w]=\frac{D_{p p}}{m^{2}} \Delta_{\xi} w+2 \lambda \nabla_{\xi} \cdot(\xi w)+\frac{2 D_{p q}}{m} \nabla_{x} \cdot\left(\nabla_{\xi} w\right)+D_{q q} \Delta_{x} w,
$$

where $w=w(t, x, \xi)$ is the (quasi)-probability distribution function. Here, $x$ and $\xi$ respectively hold for the position and velocity coordinates of the electron gas, 
and

$$
\theta_{V}[w]=\frac{i}{(2 \pi)^{3}} \int_{\mathbb{R}^{6}}\left(\frac{V\left(t, x_{+}\right)-V\left(t, x_{-}\right)}{\hbar}\right) w\left(x, \xi^{\prime}, t\right) e^{-i\left(\xi-\xi^{\prime}\right) \cdot y} d \xi^{\prime} d y
$$

is a pseudo-differential operator associated with the external potential $V$, with $x_{ \pm}=x \pm \frac{\hbar y}{2 m}$. Of course, this operator can make the quantum Fokker-Planck equation to become nonlinear in virtue of the eventual nonlinear character of the chosen potential.

One of the main aspects of dissipative theories in quantum mechanics relies on the presence of a diffusive term in the continuity equation. Indeed, if we write the balance equation for the local density, $n=\int_{\mathbb{R}^{3}} w d \xi$, we find

$$
\partial_{t} n+\nabla_{x} \cdot J=D_{q q} \Delta_{x} n, \quad \text { with } J(t, x)=\int_{\mathbb{R}^{3}} \xi w(t, x, \xi) d \xi,
$$

which is nothing else than a Fokker-Planck equation describing the time evolution of $n(t, x)$. This equation along with

$$
\partial_{t} u+\left(u \cdot \nabla_{x}\right) u=-\frac{\nabla_{x} V}{m}-\frac{\nabla_{x} \cdot \mathbb{P}}{n}-2 \lambda u-\frac{2 D_{p q}}{m}\left(\frac{\nabla_{x} n}{n}\right)+\mathcal{I}(n, u)
$$

constitute the hydrodynamic system associated with the Wigner-Fokker-Planck equation (15)-(16). Here, $u=J / n$ is the fluid mean velocity and $\mathbb{P}=E-n u \otimes u$ is the stress tensor with $E=\int_{\mathbb{R}^{3}} \xi \otimes \xi w d \xi$ denoting the kinetic energy tensor, while

$$
\mathcal{I}(n, u)=D_{q q}\left\{2\left(\frac{\nabla_{x} n}{n} \cdot \nabla_{x}\right) u+\Delta_{x} u\right\}
$$

stands for the dissipative force. The main idea underlying this derivation consists of admitting a 'classical' interpretation of the continuity equation (17) in terms of Nelsonian stochastic mechanics [37]. This theory, initiated in 1952 by I. Fényes [24], is intended to give a description of quantum mechanics by means of classical probability densities for particles undergoing Brownian motion with diffusive interactions. In this framework, the evolution of a particle subject to nondissipative Brownian motion is shown to be equivalent (in the sense of its probability and current density) to that described by the Schrödinger equation [37]. In our context we assume Brownian motion as produced by the dissipative interaction between the quantum gas and the thermal environment, the particles thus being subject to the action of forward and backward velocities $u_{+}$and $u_{-}:=u_{+}-2 u_{o}$, respectively, entering the continuity equation as

$$
\partial_{t} n+\nabla_{x} \cdot\left(n u_{ \pm}\right)= \pm D_{q q} \Delta_{x} n .
$$

Here, $u_{o}$ denotes the so-called osmotic velocity defined by

$$
u_{o}:=D_{q q} \frac{\nabla_{x} n}{n}
$$

according to Fick's law, that sets the exact balance between the osmotic current $n u_{o}$ and the diffusion current $D_{q q} \nabla_{x} n$ and somehow controls the degree 
of stochasticity of the process. Now, summing up both forward and backward Fokker-Planck equations in (19) and introducing the current mean velocity

$$
v:=\frac{1}{2}\left(u_{+}+u_{-}\right)=u_{+}-u_{o}
$$

it is easy to check that the standard continuity equation of quantum mechanics $\partial_{t} n+\nabla_{x} \cdot(n v)=0$ is recovered. Henceforth we shall use Einstein's convention (i.e. sum over repeated indices). By defining the mean backward derivative of the forward velocity as

$$
\mathcal{D}_{-} u_{+}:=\partial_{t} u_{+}+\left(u_{-} \cdot \nabla_{x}\right) u_{+}-D_{q q} \Delta_{x} u_{+},
$$

Eq. (18) can be rewritten for $u_{+}$as

$$
\mathcal{D}_{-} u_{+}=-\frac{1}{m} \nabla_{x} V-\frac{1}{n} \nabla_{x} \cdot \mathbb{P}_{u_{+}}-2 \lambda u_{+}-\frac{2 D_{p q}}{m} \frac{\nabla_{x} n}{n} .
$$

We now perform time inversion according to the following rules [26]:

$$
t \longmapsto-t, \quad \partial_{t} \longmapsto-\partial_{t}, \quad u_{ \pm} \longmapsto-u_{\mp}, \quad \mathcal{D}_{ \pm} \longmapsto-\mathcal{D}_{\mp} .
$$

Since the internal stress tensor $\mathbb{P}_{u_{+}}$is a dynamic characteristic of motion, its divergence changes sign under time inversion. Accordingly, after time inversion Eq. (20) becomes

$$
\mathcal{D}_{+} u_{-}=-\frac{1}{m} \nabla_{x} V+\frac{1}{n} \nabla_{x} \cdot \mathbb{P}_{u_{+}}+2 \lambda u_{-}-\frac{2 D_{p q}}{m} \frac{\nabla_{x} n}{n},
$$

where $\mathcal{D}_{+} u_{-}:=\partial_{t} u_{-}+\left(u_{+} \cdot \nabla_{x}\right) u_{-}+D_{q q} \Delta_{x} u_{-}$is the mean forward derivative of the backward velocity. We then sum up (20) and (21) and obtain the following frictional version of Nelson's stochastic generalization of Newton's law (in tensor notation, with $\nabla_{k}=\nabla_{x_{k}}$ )

$$
\begin{aligned}
\partial_{t} v_{j}+v_{i} \nabla_{i} v_{j}= & -\frac{1}{m} \nabla_{j}(V+\Lambda \log (n)) \\
& -D_{q q}^{2}\left[\frac{\nabla_{i} n}{n} \nabla_{i}\left(\frac{\nabla_{j} n}{n}\right)-\nabla_{j}\left(\frac{\nabla_{i}^{2} n}{n}\right)\right] .
\end{aligned}
$$

Combining now Eqs. (19) and (22) with the identity $v=u_{+}-u_{0}$, the equation for $u_{+}$

$$
\begin{aligned}
\partial_{t}\left(u_{+}\right)_{j}+\left(u_{+}\right)_{i} \nabla_{i}\left(u_{+}\right)_{j}= & -\frac{1}{m} \nabla_{j} V-\frac{\Lambda}{m} \nabla_{j} \log (n)-\frac{2 \alpha^{2}}{m \hbar^{2}} \nabla_{j} Q \\
& +D_{q q}\left[\frac{\nabla_{i} n}{n}\left(\nabla_{i}\left(u_{+}\right)_{j}-\nabla_{j}\left(u_{+}\right)_{i}\right)-\nabla_{i j}^{2}\left(u_{+}\right)_{i}\right]
\end{aligned}
$$

can be recovered. Under the original assumptions on the parameters (see for example [5] for details)

$$
\frac{1}{\omega} \ll \tau, \quad \lambda \ll \omega, \quad \omega<\frac{k_{B} T}{\hbar},
$$


$\tau$ being the characteristic time scale of the electrons, we are straightforwardly led to $\alpha \ll \hbar$, which means that the Bohm potential effects are drastically relaxed due to the spatial diffusion introduced by the quantum Fokker-Planck equation. As consequence, the $D_{q q}$ term confers 'classical' behaviour to the system at the hydrodynamic level.

Then, after the identification of the velocity as an irrotational field we get $u_{+}=\frac{1}{m} \nabla_{x} S$, hence

$$
\nabla_{j}\left(\partial_{t} S+\frac{1}{m} \nabla_{i} S \nabla_{j i}^{2} S\right)=-\nabla_{j}\left(V+\frac{2 \alpha^{2}}{\hbar^{2}} Q+\Lambda \log (n)+D_{q q} \nabla_{i i}^{2} S\right),
$$

which after formal integration along $x_{j}$ yields the following Hamilton-Jacobi type equation for the evolution of $S$ :

$$
\partial_{t} S+\frac{1}{2 m}\left|\nabla_{x} S\right|^{2}=-V-\frac{2 \alpha^{2}}{\hbar^{2}} Q-\Lambda \log (n)-D_{q q} \Delta_{x} S+\chi
$$

$\chi(t)$ being an arbitrary function of time. This along with the continuity equation

$$
\partial_{t} n+\frac{1}{m} \nabla_{x} \cdot\left(n \nabla_{x} S\right)=D_{q q} \Delta_{x} n
$$

constitute a closed potential-flow quantum hydrodynamic system, thus we may construct an 'envelope' wavefunction which contains the same physical information that the quantum Fokker-Planck equation. Indeed, if we define

$$
\psi(t, x)=\sqrt{n(t, x)} \exp \left\{\frac{i}{\alpha} S(t, x)\right\}
$$

along with the quantization rule $m \oint_{L} u_{+} d l=2 k \pi \alpha$, where $k$ is an integer and $L$ is any closed loop [43], in order to keep $\psi$ single-valued, we are led to the following Schrödinger-like equation accounting for frictional and dissipative effects

$$
i \alpha \partial_{t} \psi=H_{\alpha} \psi+\frac{\alpha^{2}}{\hbar^{2}} Q \psi+\Lambda \log (n) \psi+D_{q q}\left(\frac{i \alpha}{2} \frac{\Delta_{x} n}{n}+m \nabla_{x} \cdot \frac{J}{n}\right) \psi,
$$

where $H_{\alpha}=-\frac{\alpha^{2}}{2 m} \Delta_{x}+V$ is the electron Hamiltonian (under the new action unit $\alpha$, see [17] for details). In this picture, the magnitudes $|\psi|^{2}$ and $\frac{\alpha}{m} \operatorname{Im}\left(\bar{\psi} \nabla_{x} \psi\right)$ coincide with $n$ and $J$, respectively. Notice also that $\chi$ has been set to zero in virtue of the gauge $\widetilde{\psi}=e^{i \theta} \psi$. Here, the crossed-diffusion $D_{p q}$-term (or 'anomalous diffusion'), owing to a linear velocity-dependent frictional force caused by the interaction of the electrons with the dissipative environment, is of logarithmic type [9] (actually, $\log (n)$ can be seen as an expansion of $V$ up to $O\left(\hbar^{2}\right)$ when $V$ is assumed to be the Hartree electrostatic potential solving $\Delta_{x} V=n$ ). On the other hand, the position-diffusion $D_{q q}$-terms contain nonlinearities which form part of the Doebner-Goldin family of Schrödinger equations [22]. It is also noticeable the fact that the term involving $D_{p p}=2 m \lambda k_{B} T$, responsible for the decoherence process, does not contribute to the final form of Eq. (3). This is due to the fact that the moment system has been truncated at the level of the 
momentum equation, while the $D_{p p}$-contribution is only 'visible' at the next level, i.e. that of the energy equation. However, the role played by $D_{p p}$ is essential for the fulfillment of the uncertainty inequality as well as for the Lindblad form [33] of the Wigner-Fokker-Planck equation, thus for the positivity preservation of the density matrix operator. As a matter of fact, a sufficient and necessary condition to fit Lindblad's class is that the reservoir parameters be such that the inequality $D_{p p} D_{q q}-D_{p q}^{2} \geq \hbar^{2} \lambda^{2} / 4$ holds.

\section{ON THE EXISTENCE OF A REgUlar ARGUMENT FUnCTION}

The right definition and regularity of the argument of a complex wavefunction $\psi$ has been one of the most serious drawbacks in quantum mechanics for years, when aiming to connect the Schrödinger equation with the fluid description provided by the balance laws for the square amplitude $n_{\psi}=|\psi|^{2}$ and the quantum phase $S_{\psi}$ via the Madelung transformation [43]. To the best of our knowledge, this is the first time in which an analytical treatment partially ends up with this question and make it possible to rigorously write the Madelung form of the wavefunction in an unique way (up to additive constant factors). This section is devoted to discuss several rigorous aspects related to the existence of $S_{\psi}$ as well as to find a smooth argument for $\psi$ under just some regularity assumptions for $\nabla_{x} \psi / \psi$, which are known to hold true in $H_{\delta}^{2}$.

In [30] a very general approach to this sort of nonlinear Schrödinger problems stemming from quantum hydrodynamics was carried over to solve a flow-potential quantum hydrodynamical system by using its formal equivalence with the equation

$$
i \hbar \partial_{t} \psi=H \psi+h\left(n_{\psi}\right) \psi+S_{\psi} \psi,
$$

which contains Kostin's term $S_{\psi} \psi$ [31] describing Schrödinger-Langevin dynamics, where $H=-\frac{\hbar^{2}}{2 m} \Delta_{x}+V(x)$ stands for the electron Hamiltonian and where the enthalpy function $h$ is defined by

$$
h^{\prime}(r)=\frac{p^{\prime}(r)}{r} \quad \forall r>0, \quad h(1)=0,
$$

the scalar pressure $p$ (typically expressed as $p\left(n_{\psi}\right)=\theta n_{\psi}^{\gamma}$ in classical fluid dynamics with $\theta>0$ standing for the temperature) being assumed to depend only on the particle density $n_{\psi}$. In the particular case $\gamma=1$ the isothermal fluid condition $p=\theta n_{\psi}$ is met, which is straightforwardly translated into the nonlinear term $\theta \log \left(|\psi|^{2}\right) \psi$ in the Schrödinger picture. In [30] it is already shown the local existence of $H_{\delta}^{2}$ solutions to a family of logarithmic Schrödinger equations with Poisson coupling in bounded domains. Nonetheless, some mathematical shortcomings are still present in the whole theory concerning the selection of an argument $S_{\psi}=-i \log (\psi /|\psi|)$ owing to the mutivaluedness of the complex logarithm. As a matter of fact, in [30] the eventual ambiguity of the argument 
is partially overcome by introducing it as the solution of the following elliptic problem

$$
\begin{aligned}
\Delta_{x} S_{\psi} & =\hbar \operatorname{Im}\left(\nabla_{x} \cdot \frac{\nabla_{x} \psi}{\psi}\right) \\
& =\hbar \frac{n_{\psi} \operatorname{Im}\left(\bar{\psi} \Delta_{x} \psi\right)-2 \operatorname{Re}\left(\bar{\psi} \nabla_{x} \psi\right) \cdot \operatorname{Im}\left(\bar{\psi} \nabla_{x} \psi\right)}{n_{\psi}^{2}}
\end{aligned}
$$

with somehow arbitrary Dirichlet boundary values, far from vacuum regions $\left(n_{\psi}=0\right)$ in $\Omega$ in order to circumvent the singularities. In spite of that, some important defficiencies must be still managed to be fully rigorous from a mathematical point of view. To illustrate this point, we can consider for instance $\Omega=B_{1} \subset \mathbb{R}^{3}$ the unit ball centered at the origin and $\psi(x)=\exp \left\{\frac{i}{2 \alpha}|x|^{2}\right\}$ for all $x \in \Omega$. Then, it becomes clear that $S_{\psi}(x)=|x|^{2} / 2$ is a global argument for $\psi$ that satisfies $\Delta S=3$ in $\Omega$. On the contrary, solving the problem

$$
\Delta \sigma=3 \text { in } \Omega, \quad \sigma \equiv 1 \text { in } \partial \Omega,
$$

yields $\sigma(x)=\frac{1}{2}\left(|x|^{2}+1 /|x|^{2}\right)$, which clearly is not an argument for $\psi$. Of course, this means that not every solution to Eq. (25) with prescribed values on the boundary of $\Omega$ is a 'true argument' of the wavefunction. In this context, Neumann boundary conditions seem to be more appropriate given that $\psi$ fully determines $\nabla_{x} S_{\psi}$ through the physical observables $n_{\psi}$ and $J_{\psi}$. Indeed, if we assume that a pure quantum state can be decomposed in Madelung's modulus-argument form (10), then the equation

$$
\nabla_{x} S_{\psi}=\alpha \operatorname{Im}\left(\frac{\nabla_{x} \psi}{\psi}\right)=m\left(\frac{J_{\psi}}{n_{\psi}}\right)
$$

is formally fulfilled for the gradient velocity field, which allows for a connection between the hydrodynamic and the Schrödinger descriptions of quantum mechanics. What we propose here is to directly tackle Eq. (26), as it readily implies Eq. (25) (by just taking its divergence) and moreover does not 'see' eventual solutions of (25) which are not 'true arguments'. In this spirit, the problem is reduced to the computation of a scalar potential associated with $\operatorname{Im}\left(\nabla_{x} \psi / \psi\right)$. This obviously requires the irrotationality of the field, which can be deduced from Schwartz's Lemma and the fact that the domain is simply-connected, as shown in [1,2] in a much more general situation. Proceeding like this, we shall obtain an unique (up to an additive constant) solution $S_{\psi}$ to Eq. (26) for a given $\psi \in H_{\delta}^{2}$, that of course also solves the corresponding Neumann boundary value problem associated with Eq. (25). Thus, a countable family of functions $S_{\psi}^{l} \in H^{2}(\Omega), l \in \mathbb{Z}$, there exists such that

$$
\begin{aligned}
\psi(x) & =\sqrt{n_{\psi}(x)} \exp \left\{\frac{i}{\alpha} S_{\psi}^{l}(x)\right\}, \quad \text { a.e. } x \in \Omega, \\
S_{\psi}^{l}-S_{\psi}^{m} & =2 \pi \alpha(l-m) .
\end{aligned}
$$


Furthermore, for any fixed $\mu \in \mathbb{R}$, the mapping $\psi \mapsto S_{\psi}$ is continuous from $H_{\delta}^{2}$ to $\left\{S \in H^{2}(\Omega): \int_{\Omega} S d x=\mu\right\}$. Indeed, this makes it possible to find a continuousin-time argument for any given $\psi \in X_{\delta}^{T}$ solution to a general Schrödinger-like equation.

A different approach to the treatment of $S_{\psi}$ was recently given in [3], where the authors demonstrate the existence of finite energy solutions to the quantum hydrodynamic system (coupled to Poisson's potential $V$ )

$$
\begin{gathered}
\partial_{t} n_{\psi}+\nabla_{x} \cdot J_{\psi}=0 \\
\partial_{t} J_{\psi}+\nabla_{x} \cdot\left(\frac{J_{\psi} \otimes J_{\psi}}{n_{\psi}}\right)+J_{\psi}+\nabla_{x} p\left(n_{\psi}\right)=-n_{\psi} \nabla_{x}(V+m Q)
\end{gathered}
$$

via its formal equivalence with the family of nonlinear Schrödinger equations (24). However, they do not have the need to calculate $S_{\psi}$ thanks to the use of a fractional step method which allows them to obtain the macroscopic magnitudes, say $n_{\psi}$ and $J_{\psi}$, by just solving the previous Schrödinger-Poisson equation without the Schrödinger-Langevin term $S_{\psi} \psi$ and taking the adequate limit. On the contrary, computing the argument of the wavefunction seems to be unavoidable for us to be able to establish the potential-flow hydrodynamic system associated with our Schrödinger equations.

The simple connectedness of the domain will be of crucial importance for our purposes, since otherwise we are oblied to admit jumps in $S_{\psi}$. The following simple example so testifies it: take the ringed cylinder

$$
\Omega=\left\{(x, y, x) \in \mathbb{R}^{3}: 0<r^{2}<x^{2}+y^{2}<1,0<z<1\right\}
$$

and consider the complex function $\psi: \Omega \rightarrow \mathbb{C}$ defined as $\psi(x, y, z)=x+i y$. It is clear that $\psi \in C^{\infty}(\Omega) \cap L^{2}(\Omega)$ and $|\psi|=\sqrt{x^{2}+y^{2}}>r>0$ for all $(x, y, z) \in$ $\Omega$, but $\psi$ has not a continuous argument $S_{\psi}$ in $\Omega$. Indeed, if such a function $S_{\psi}: \Omega \rightarrow \mathbb{R}$ could be built up, we might consider $\mathcal{R}\left(0 ; r^{2}, 1\right) \subset \mathbb{C}$ the annulus centered at zero with radius $r^{2}$ and 1 and $i: \mathcal{R}\left(0 ; r^{2}, 1\right) \rightarrow \Omega$ defined by $i(w)=$ $(\operatorname{Re}(w), \operatorname{Im}(w), 0)$ in such a way that $S_{\psi} \circ i$ would be a continuous argument for the identity operator $\psi \circ i: \mathcal{R}\left(0 ; r^{2}, 1\right) \rightarrow \mathcal{R}\left(0 ; r^{2}, 1\right)$. However, this is impossible in virtue of well known results from complex analysis (see for instance Theorem 13.18 in $[40])$.

In the sequel we are concerned with the problem of existence of a regular argument for a strong solution of a Schrödinger equation. The first step is to solve Eq. (26) for a given wavefunction $\psi$. To proceed we shall follow the track of some ideas developed in [1] and [2].

Lemma 3.1. Let $\Omega \subset \mathbb{R}^{3}$ be a simply-connected, Lipschitz-continuous bounded domain and $0 \leq k \in \mathbb{Z}$. Then the following assertions hold.

(i) For all complex functions $\psi \in H^{k}(\Omega)$ such that

$$
\frac{\nabla \psi}{\psi} \in\left(H^{k-1}(\Omega)\right)^{3}, \quad \frac{(\nabla \otimes \nabla) \psi}{\psi}, \frac{\nabla \psi}{\psi} \otimes \frac{\nabla \psi}{\psi} \in\left(H^{k-2}(\Omega)\right)^{9},
$$


WELLPOSEDNESS OF LOG. SCHRÖDINGER EQ. OF DOEBNER-GOLDIN TYPE 13

there exists an unique (up to an additive constant) function $S_{\psi} \in H^{k}(\Omega)$ that solves Eq. (26). Besides, given $\mu \in \mathbb{R}$ there exists an unique $S_{\psi} \in$ $H^{k}(\Omega)$ solution to Eq. (26) such that $\int_{\Omega} S_{\psi} d x=\mu$, and also an unique $\beta_{\mu} \in[0,2 \pi \alpha)$ such that the family

$$
S_{\psi}^{l}:=S_{\psi}+\beta_{\mu}+2 \pi l \alpha, \quad l \in \mathbb{Z},
$$

satisfies (27)-(28).

(ii) Under the hypotheses of (i), there exists $C>0$ such that

$$
\left\|S_{\psi}-S_{\phi}\right\|_{H^{k}(\Omega)} \leq C\left\|\operatorname{Im}\left(\frac{\nabla \psi}{\psi}\right)-\operatorname{Im}\left(\frac{\nabla \phi}{\phi}\right)\right\|_{H^{k-1}(\Omega)}
$$

for all $S_{\psi}, S_{\phi}$ solutions to Eq. (26) (associated with $\psi$ and $\phi$, respectively) satisfying $\int_{\Omega} S_{\psi} d x=\int_{\Omega} S_{\phi} d x$.

This result is mainly achieved by using Proposition 2.10 in [2] and Theorem 1 in [1], that we state below for selfconsistency.

Proposition 3.1 (Amrouche-Girault [2]). Let $\Omega$ be a bounded Lipschitzcontinuous domain of $\mathbb{R}^{d}, m$ an integer and $r$ any real number with $1<r<\infty$.

(i) If $p \in \mathcal{D}^{\prime}(\Omega)$ has its gradient in $W^{m-1, r}(\Omega)$, then $p$ belongs to $W^{m, r}(\Omega)$. If in addition $\Omega$ is connected, then there exists a constant $C>0$ such that the following inequality is satisfied

$$
\forall[p] \in W^{m, r}(\Omega) / \mathbb{R}, \quad\|[p]\|_{W^{m, r}(\Omega) / \mathbb{R}} \leq C\|\nabla p\|_{W^{m-1, r}(\Omega)} .
$$

If $\Omega$ is arbitrary (not necessarily bounded nor Lipschitz-continuous), then $p$ belongs to $W_{l o c}^{m, r}(\Omega)$.

(ii) When $m \geq 0$ and $\Omega$ is connected, there exists a constant $C>0$ such that all distributions $p$ in $\mathcal{D}^{\prime}(\Omega)$ with $\nabla p$ in $W^{m-1, r}(\Omega)$ and $\int_{\Omega} p d x=0$ satisfy the bound

$$
\|p\|_{W^{m, r}(\Omega)} \leq C\|\nabla p\|_{W^{m-1, r}(\Omega)} .
$$

Theorem 3.1 (Amrouche-Ciarlet-Ciarlet [1]). Let $f \in\left(H^{-m}(\Omega)\right)^{3}$ for some integer $m \geq 0$. Then, the following assertions are equivalent:

(i) $H^{-m}(\Omega)\langle f, \varphi\rangle_{H_{0}^{m}(\Omega)}=0$ for any $\varphi \in V_{m}=\left\{\varphi \in\left(H_{0}^{m}(\Omega)\right)^{3}: \nabla \cdot \varphi=0\right\}$.

(ii) $H^{-m}(\Omega)\langle f, \varphi\rangle_{H_{0}^{m}(\Omega)}=0$ for any $\varphi \in V=\left\{\varphi \in(\mathcal{D}(\Omega))^{3}: \nabla \cdot \varphi=0\right\}$.

(iii) There exists a distribution $\chi \in H^{-m+1}(\Omega)$, unique up to an additive constant, such that $f=\nabla \chi$ in $\Omega$.

If $\Omega$ is in addition simply-connected, then the three previous statements are equivalent to:

(iv) $\operatorname{curl}(f)=0$ in $\Omega$. 
Proof of Lemma 3.1. Let $\psi \in H^{k}(\Omega)$ be a complex function under the hypotheses stated in (i). Then

$$
\nabla \otimes\left\{\operatorname{Im}\left(\frac{\nabla \psi}{\psi}\right)\right\}=\operatorname{Im}\left(\frac{(\nabla \otimes \nabla) \psi}{\psi}-\frac{\nabla \psi}{\psi} \otimes \frac{\nabla \psi}{\psi}\right),
$$

hence $\operatorname{curl}(\operatorname{Im}(\nabla \psi / \psi))=0$ in the sense of distributions. By Theorem 3.1 $\left((\right.$ iv $) \Rightarrow($ iii $)$ ) we deduce the existence of $S_{\psi} \in \mathcal{D}^{\prime}(\Omega)$, unique up to an additive constant, such that $\nabla S_{\psi}=\alpha \operatorname{Im}(\nabla \psi / \psi)$, that is to say it solves Eq. (26). Using now the fact that $\nabla \psi / \psi \in\left(H^{k-1}(\Omega)\right)^{3}$ by hypothesis along with Proposition 3.1 (i), we find that $S_{\psi} \in H^{k}(\Omega)$. In addition, given $\mu \in \mathbb{R}$ and $S \in H^{k}(\Omega)$ any solution to Eq. (26), we find that $S_{\psi}$ defined by

$$
S_{\psi}(x):=S(x)+\frac{1}{|\Omega|}\left(\mu-\int_{\Omega} S d x\right)
$$

is the unique solution to Eq. (26) that satisfies $\int_{\Omega} S_{\psi} d x=\mu$. Finally, if defining

$$
\psi^{S}(x):=|\psi(x)| \exp \left\{\frac{i}{\alpha} S_{\psi}(x)\right\} \text { a.e. } x \in \Omega,
$$

we can easily deduce that $\psi^{S} \in H^{k}(\Omega)$ in virtue of

$$
\nabla \psi^{S}=\left\{\operatorname{Re}\left(\frac{\nabla \psi}{\psi}\right)+i \operatorname{Im}\left(\frac{\nabla \psi}{\psi}\right)\right\} \psi^{S},
$$

thus $\psi \nabla \psi^{S}=\psi^{S} \nabla \psi$. As consequence $\nabla\left(\psi / \psi^{S}\right)=0$, which implies the existence of an unitary $z \in \mathbb{C}$ such that $\psi / \psi^{S} \equiv z$. Therefore, there exists $\beta \in \mathbb{R}$ such that $\psi=e^{i \beta / \alpha} \psi^{S}$. In particular,

$$
\psi(x)=|\psi(x)| \exp \left\{\frac{i}{\alpha}\left(S_{\psi}(x)+\beta\right)\right\} \quad \text { a.e. } x \in \Omega .
$$

Of course $\beta$ is not unique, as whether $\beta_{1}, \beta_{2} \in \mathbb{R}$ are chosen so as to satisfy Eq. (30), then it is clear that $\beta_{1}-\beta_{2}=2 \pi \alpha l$ for some $l \in \mathbb{Z}$. Now, it is enough to choose $\beta_{\mu}$ as the unique number in $[0,2 \pi \alpha)$ fulfilling (30). This concludes the proof of (i).

In virtue of Proposition 3.1 (ii), we have that a positive constant $C$ does exist such that

$$
\|S\|_{H^{k}(\Omega)} \leq C\|\nabla S\|_{H^{k-1}(\Omega)}
$$

for all $S \in H^{k}(\Omega)$ with vanishing mean value. Therefore, if $S_{\psi}, S_{\phi}$ are two solutions to Eq. (26) as those given in (i), then it is clear that $S_{\psi}-S_{\phi} \in H^{k}(\Omega)$ and $\int_{\Omega}\left(S_{\psi}-S_{\phi}\right) d x=0$, so that $\left\|S_{\psi}-S_{\phi}\right\|_{H^{k}(\Omega)} \leq C\left\|\nabla\left(S_{\psi}-S_{\phi}\right)\right\|_{H^{k-1}(\Omega)}$. This ends the proof.

\section{Remark 3.1. Notice that}

(i) We need $\Omega$ to be connected in order that Proposition 3.1 can be applied, yet otherwise a parallel argument might be carried out on every connected component. 
(ii) Our main assumption in Theorem 1.1, i.e. the fact that

$$
\operatorname{ess-inf}\left\{\left|\psi_{0}(x)\right|: x \in \Omega\right\}>\delta,
$$

implies the conditions of Lemma 3.1

The following result establishes the main regularity properties of the fundamental observables we are concerned with. In the sequel we shall skip $\Omega$ from the subsequent norms for the sake of notational simplicity, and write $\|\cdot\|_{X}$ instead of $\|\cdot\|_{X(\Omega)}$ for any given functional space $X$.

Lemma 3.2. Let $\Omega \subset \mathbb{R}^{3}$ be a $C^{1}$ bounded domain, $\delta>0$ and $\psi \in H_{\delta}^{2}$. Then, for $n=|\psi|^{2}$ and $J=\frac{\alpha}{m} \operatorname{Im}(\bar{\psi} \nabla \psi)$, the following identity holds

$$
\bar{\psi} \nabla \psi=\frac{1}{2} \nabla n+\frac{m i}{\alpha} J .
$$

As consequence

(i) $\sqrt{n} \in H_{\delta}^{2}, n \in H_{\delta^{2}}^{2}, J, \nabla n / n, J / n \in\left(H^{1}(\Omega)\right)^{3}$, and the mappings

$$
\psi \mapsto \sqrt{n}, n, J, \frac{\nabla n}{n}, \frac{J}{n}
$$

are continuous from $H_{\delta}^{2}$ onto the corresponding functional space in each case.

(ii) $\Delta \psi \in L^{2}(\Omega)$ may be written as

$$
\Delta \psi=\left\{-\frac{2 m}{\hbar^{2}} Q-\frac{m}{\alpha}\left(\frac{m}{\alpha} \frac{|J|^{2}}{n^{2}}-i \frac{\nabla \cdot J}{n}\right)\right\} \psi,
$$

where $Q$ is given by formula (8).

Proof. The identity (31) comes out straightforwardly from the definition of $J$ and the fact that $\nabla n=2 \operatorname{Re}(\bar{\psi} \nabla \psi)$. To check (i) we first notice that the regularity of $\sqrt{n}$ and its continuous dependence upon $\psi$ follow from the fact that $\sqrt{n}=$ $|\psi|$. Also, $\psi \in L^{\infty}(\Omega)$ as sheds from the Sobolev embedding $H^{2}(\Omega) \hookrightarrow L^{\infty}(\Omega)$. Furthermore, $\bar{\psi} \nabla \psi \in L^{2}(\Omega)$ and

$$
\nabla \otimes(\bar{\psi} \nabla \psi)=\nabla \bar{\psi} \otimes \nabla \psi+\bar{\psi}(\nabla \otimes \nabla) \psi \in L^{2}(\Omega),
$$

since $\nabla \psi \in H^{1}(\Omega) \subset L^{4}(\Omega)$, so that $\nabla \bar{\psi} \otimes \nabla \psi \in L^{2}(\Omega)$. Hence $\bar{\psi} \nabla \psi \in\left(H^{1}(\Omega)\right)^{3}$. Taking real parts in $(31)$ we find that $\nabla n \in\left(H^{1}(\Omega)\right)^{3}$, thus $n \in H^{2}(\Omega)$. Also, the nonvacuum condition $|\psi|>\delta$ implies that $n \in H_{\delta^{2}}^{2}$. Taking now imaginary parts we can easily deduce that $J \in\left(H^{1}(\Omega)\right)^{3}$. Again, the fact that $|\psi|>\delta$ allows to guarantee that $\nabla n / n, J / n \in\left(H^{1}(\Omega)\right)^{3}$.

To observe the continuity of the mappings stated in (i) it is enough to show that for all $\psi, \phi \in H_{\delta}^{2}$ we have

$$
\left\|n_{\psi}-n_{\phi}\right\|_{L^{2}} \leq C\left\|n_{\psi}-n_{\phi}\right\|_{L^{\infty}}=C\|\psi \bar{\psi}-\phi \bar{\phi}\|_{L^{\infty}}
$$




$$
\begin{aligned}
\|\bar{\psi} \nabla \psi-\bar{\phi} \nabla \phi\|_{L^{2}} & \leq\|\bar{\psi}(\nabla \psi-\nabla \phi)\|_{L^{2}}+\|\nabla \phi(\bar{\psi}-\bar{\phi})\|_{L^{2}} \\
& \leq\|\psi\|_{L^{\infty}}\|\nabla \psi-\nabla \phi\|_{L^{2}}+\|\nabla \phi\|_{L^{2}}\|\psi-\phi\|_{L^{\infty}} \\
& \leq C\left(\|\psi\|_{H^{2}}+\|\phi\|_{H^{2}}\right)\|\psi-\phi\|_{H^{2}},
\end{aligned}
$$

$\|\nabla \otimes(\bar{\psi} \nabla \psi-\bar{\phi} \nabla \phi)\|_{L^{2}} \leq\|\nabla \bar{\psi} \otimes(\nabla \psi-\nabla \phi)\|_{L^{2}}+\|\nabla \phi \otimes(\nabla \bar{\psi}-\nabla \bar{\phi})\|_{L^{2}}$

$$
\begin{aligned}
& +\|\bar{\psi} \nabla \otimes \nabla(\psi-\phi)\|_{L^{2}}+\|(\bar{\psi}-\bar{\phi}) \nabla \otimes \nabla \phi\|_{L^{2}} \\
\leq & C\left(\|\psi\|_{H^{2}}+\|\phi\|_{H^{2}}\right)\|\psi-\phi\|_{H^{2}},
\end{aligned}
$$

where we denoted $C$ various positive constants only depending on $\Omega$. Therefore the mapping $\psi \in H_{\delta}^{2} \mapsto \bar{\psi} \nabla \psi \in H^{1}(\Omega)$ is continuous, thus $\psi \mapsto \nabla n$ and $\psi \mapsto J$ so are. This along with (32) yield the continuity of $H_{\delta}^{2} \ni \psi \mapsto n \in H_{\delta^{2}}^{2}$. Next, checking that $\psi \mapsto \nabla \psi / \psi$ is continuous from $H_{\delta}^{2}$ onto $L^{2}(\Omega)$ is a simple matter stemming from the strict positivity of $|\psi|$. Furthermore, we have

$$
\left\|\nabla \otimes\left(\frac{\nabla \psi}{\psi}-\frac{\nabla \phi}{\phi}\right)\right\|_{L^{2}} \leq\left\|\frac{\nabla \otimes \nabla \psi}{\psi}-\frac{\nabla \otimes \nabla \phi}{\phi}\right\|_{L^{2}}+\left\|\frac{\nabla \psi}{\psi} \otimes \frac{\nabla \psi}{\psi}-\frac{\nabla \phi}{\phi} \otimes \frac{\nabla \phi}{\phi}\right\|_{L^{2}},
$$

where the first term in the right-hand side is bounded as in (34) by

$$
\begin{aligned}
\frac{1}{\delta^{2}}\|\phi \nabla \otimes \nabla \psi-\psi \nabla \otimes \nabla \phi\|_{L^{2}} & \leq \frac{1}{\delta^{2}}\left(\|\phi\|_{L^{\infty}}\|\psi-\phi\|_{H^{2}}+\|\phi\|_{H^{2}}\|\psi-\phi\|_{L^{\infty}}\right) \\
& \leq \frac{C}{\delta^{2}}\|\phi\|_{H^{2}}\|\psi-\phi\|_{H^{2}},
\end{aligned}
$$

while the second one is bounded by

$\frac{1}{\delta^{4}}\left\|\phi^{2} \nabla \psi \otimes \nabla \psi-\psi^{2} \nabla \phi \otimes \nabla \phi\right\|_{L^{2}}$

$\leq \frac{C}{\delta^{4}}\left\{\|\phi\|_{L^{\infty}}^{2}\left(\|\nabla \phi\|_{L^{4}}+\|\nabla \psi\|_{L^{4}}\right)\|\nabla \psi-\nabla \phi\|_{L^{4}}+\|\nabla \phi\|_{L^{4}}^{2}\|\psi+\phi\|_{L^{\infty}}\|\psi-\phi\|_{L^{\infty}}\right\}$ (36)

$\leq \frac{C}{\delta^{4}}\|\phi\|_{H^{2}}^{2}\left(\|\phi\|_{H^{2}}+\|\psi\|_{H^{2}}\right)\|\psi-\phi\|_{H^{2}}$.

Consequently, we have that $H_{\delta}^{2} \ni \psi \mapsto \nabla \psi / \psi \in H^{1}(\Omega)$ is also continuous. Finally, taking real and imaginary parts we see that both $\psi \mapsto \nabla n / n$ and $\psi \mapsto$ $J / n$ are continuous from $H_{\delta}^{2}$ onto $H^{1}(\Omega)$. This proves the first statement of the lemma. 
To demonstrate (ii) we use that

$$
\nabla \psi=\left(\frac{\nabla n}{2 n}+\frac{m i}{\alpha} \frac{J}{n}\right) \psi \in H^{1}(\Omega)
$$

which is a straightforward consequence of formula (31) and of the previous estimates. Then, by taking divergences we achieve the expression in (ii) for $\Delta \psi$ after simple calculus.

We now make explicit the rigorous connection between the wavefunction description of the quantum-mechanical process and its associated hydrodynamic formulation.

Lemma 3.3. Let $\Omega \subset \mathbb{R}^{3}$ be a bounded domain, $\delta>0, T>0$, and let $\psi \in X_{\delta}^{T}$ be a strong solution of the following model Schrödinger equation

$$
i \partial_{t} \psi=-\frac{\alpha}{2 m} \Delta_{x} \psi+\Theta[n, J] \psi,
$$

where $\Theta: H_{\delta^{2}}^{2} \times\left(H^{1}(\Omega)\right)^{3} \rightarrow L^{2}(\Omega)$ is a continuous (complex) operator. Then

$$
n \in X_{\delta^{2}}^{T}, \quad \frac{\nabla_{x} n}{n}, J, \frac{J}{n} \in C\left([0, T) ; H^{1}(\Omega)\right) \cap C^{1}\left([0, T) ; H^{-1}(\Omega)\right),
$$

and the following identities hold:

(i) $\partial_{t} n+\nabla_{x} \cdot J=2 \operatorname{Im}(\Theta[n, J]) n$ in a strong sense.

(ii) $\partial_{t} J=\frac{\alpha^{2}}{2 m^{2}} \operatorname{Re}\left(\psi^{2} \nabla_{x}\left(\frac{\Delta_{x} \bar{\psi}}{\psi}\right)\right)-\frac{\alpha}{m} \operatorname{Re}\left(\psi^{2} \nabla_{x}\left(\frac{\overline{\Theta[n, J] \psi}}{\psi}\right)\right)$ in the sense of distributions.

(iii) $\partial_{t}\left(\frac{J}{n}\right)=-\nabla_{x}\left(\frac{\alpha^{2}}{\hbar^{2} m} Q+\frac{|J|^{2}}{2 n^{2}}+\frac{\alpha}{m} \operatorname{Re}(\Theta[n, J])\right)$ in the sense of distributions.

Proof. The regularities of $n, J, \nabla_{x} n / n$ and $J / n$ are deduced from the previous lemma and the fact that $t \mapsto \psi(t)$ is a continuous mapping in $H^{2}(\Omega)$.

(i) follows after multiplying the Schrödinger equation by $-i \bar{\psi}$ and taking twice the real part. As $t \mapsto \Theta[n, J](t) n(t)$ as well as $t \mapsto \nabla_{x} \cdot J(t)$ are continuous from $[0, T)$ onto $L^{2}(\Omega)$, we find that $n \in C^{1}\left([0, T) ; L^{2}(\Omega)\right)$.

We now prove (ii). It is a simple task to verify that $\partial_{t} J=\frac{\alpha}{m} \operatorname{Im}\left(\partial_{t} \bar{\psi} \nabla_{x} \psi+\right.$ $\left.\bar{\psi} \partial_{t} \nabla_{x} \psi\right)$ in the sense of distributions. Using Eq. (38) this identity can be rewritten as

$\partial_{t} J=\frac{\alpha^{2}}{2 m^{2}} \operatorname{Re}\left(\bar{\psi} \nabla_{x} \Delta_{x} \psi-\nabla_{x} \psi \Delta_{x} \bar{\psi}\right)+\frac{\alpha}{m} \operatorname{Re}\left(\nabla_{x} \psi \overline{\Theta[n, J] \psi}-\bar{\psi} \nabla_{x}(\Theta[n, J] \psi)\right)$,

which can be easily recast in the form stated in (ii). Note that the regularity of $\psi$ and $\Theta[n, J]$ allows $\partial_{t} J$ to make sense in $H^{-1}(\Omega)$.

According to the previous balance laws for $n$ and $J$ we get

$$
\partial_{t}\left(\frac{J}{n}\right)=\frac{\alpha}{m}\left\{\frac{\alpha}{2 m} \operatorname{Re}\left(\frac{\psi}{\bar{\psi}} \nabla_{x}\left(\frac{\Delta_{x} \bar{\psi}}{\psi}\right)\right)-\operatorname{Re}\left(\frac{\psi}{\bar{\psi}} \nabla_{x}\left(\frac{\overline{\Theta[n, J] \psi}}{\psi}\right)\right)\right\}
$$




$$
+\frac{J}{n}\left\{\frac{\nabla_{x} \cdot J}{n}-2 \operatorname{Im}(\Theta[n, J])\right\} .
$$

The subsequent computation of $\frac{\psi}{\bar{\psi}} \nabla_{x}\left(\frac{\Delta_{x} \bar{\psi}}{\psi}\right)$ via Lemma 3.2 (ii) and that of $\frac{\psi}{\psi} \nabla_{x}\left(\frac{\overline{\Theta[n, J] \psi}}{\psi}\right)$ gives

$$
\begin{aligned}
\frac{\psi}{\bar{\psi}} \nabla_{x}\left(\frac{\Delta_{x} \bar{\psi}}{\psi}\right)= & \nabla_{x}\left(\frac{\Delta_{x} n}{2 n}-\frac{\left|\nabla_{x} n\right|^{2}}{4 n^{2}}\right) \\
& -\frac{m}{\alpha}\left\{\frac{m}{\alpha} \nabla_{x}\left(\frac{|J|^{2}}{n^{2}}\right)+i \nabla_{x}\left(\frac{\nabla_{x} \cdot J}{n}\right)\right\} \\
& -\frac{2 i}{n^{2}} \operatorname{Im}\left(\bar{\psi} \nabla_{x} \psi\right)\left\{\frac{\Delta_{x} n}{2}-\frac{\left|\nabla_{x} n\right|^{2}}{4 n}-\frac{m^{2}}{\alpha^{2}} \frac{|J|^{2}}{n}-\frac{m i}{\alpha} \nabla_{x} \cdot J\right\}, \\
\frac{\psi}{\bar{\psi}} \nabla_{x}\left(\frac{\overline{\Theta[n, J] \psi}}{\psi}\right)= & \nabla_{x}(\overline{\Theta[n, J]})+\overline{\Theta[n, J]}\left(\frac{\nabla_{x} \bar{\psi}}{\bar{\psi}}-\frac{\nabla_{x} \psi}{\psi}\right),
\end{aligned}
$$

respectively. Finally, taking real parts and making some simplifications we arrive at the equation shown in (iii). Furthermore, using the regularity known for $\psi$ and $\Theta[n, J]$ it is easily found that $J / n, \nabla_{x} n / n \in\left(C^{1}\left([0, T) ; H^{-1}(\Omega)\right)\right)^{3}$.

To close this section, we shall prove that strong solutions of a general Schrödinger equation as that of Lemma 3.3 can be rigorously decomposed in Madelung's form in an unique way (up to a change of constant global phase) in terms of a regular argument.

Theorem 3.2. Let $\Omega \subset \mathbb{R}^{3}$ be a simply-connected, $C^{1}$ bounded domain. Let also $\delta>0, T>0$, and $\psi \in X_{\delta}^{T}$ under the hypotheses of Lemma 3.3 Then, there exists $S_{\psi} \in X^{T}$ such that the following properties are accomplished.

(i) For any fixed $t \in[0, T)$, the equation $\nabla_{x} S_{\psi}(t)=\frac{J}{n}(t)$ holds almost everywhere in $\Omega$ and

$$
\psi(0, x)=\sqrt{n(0, x)} \exp \left\{\frac{i}{\alpha} S_{\psi}(0, x)\right\} .
$$

(ii) The evolution equation

$$
\partial_{t} S_{\psi}=-\frac{\alpha^{2}}{\hbar^{2}} Q-\frac{m}{2} \frac{|J|^{2}}{n^{2}}-\alpha \operatorname{Re}(\Theta[n, J])
$$

holds strongly in $[0, T)$.

(iii) There exists a countable family $\left\{S_{\psi}^{l}\right\}_{l \in \mathbb{Z}} \subset X^{T}$ such that the Madelung decomposition

$$
\psi(t, x)=\sqrt{n(t, x)} \exp \left\{\frac{i}{\alpha} S_{\psi}^{l}(t, x)\right\}
$$

holds for any $l \in \mathbb{Z}$.

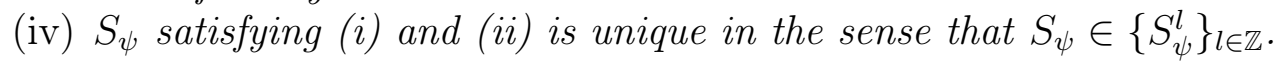


Proof. Define $K: H_{\delta^{2}}^{2} \times\left(H^{1}(\Omega)\right)^{3} \rightarrow L^{2}(\Omega)$ as

$$
K[n, J]:=\frac{\alpha^{2}}{\hbar^{2}} Q+\frac{m}{2} \frac{|J|^{2}}{n^{2}}+\alpha \operatorname{Re}(\Theta[n, J]) .
$$

On one hand, the fact that $|\psi|$ is nonvanishing along with the Sobolev embedding $H^{2}(\Omega) \hookrightarrow L^{\infty}(\Omega)$ makes $t \mapsto K\left[n_{\psi}, J_{\psi}\right](t)$ continuous from $[0, T)$ onto $L^{2}(\Omega)$. On the other hand, $\psi(0) \in H_{\delta}^{2}$. Then, by fixing (for instance) $\mu=0$ in Eq. (29) the hypotheses of Lemma 3.1 (i) are fulfilled, hence there exist $S_{\psi(0)} \in H^{2}(\Omega)$ and $\beta_{0} \in[0,2 \pi \alpha)$ such that

$$
\psi(0, x)=\sqrt{n(0, x)} \exp \left\{\frac{i}{\alpha} S_{0}(x)\right\} \quad \text { a.e. } x \in \Omega,
$$

where we denoted $S_{0}=S_{\psi(0)}+\beta_{0}$. Now we can define

$$
S_{\psi}(t, x):=S_{0}(x)-\int_{0}^{t} K[n, J](s, x) d s+\frac{1}{|\Omega|} \int_{\Omega} \int_{0}^{t} K[n, J](s, y) d s d y
$$

for all $t \in[0, T)$. The fact that the operators $\Theta$ and $K$ depend upon $\psi$ only through the observables $n$ and $J$ emerges here as a relevant feature to make the definition of $S_{\psi}$ explicit. Let us finally check that $S_{\psi}$ defined as above satisfies (i)-(iv).

The definition of $S_{\psi}$ jointly with (41) yield (39). Also, the continuity of $t \mapsto K\left[n_{\psi}, J_{\psi}\right](t)$ in $L^{2}(\Omega)$ implies that $S_{\psi} \in C^{1}\left((0, T) ; L^{2}(\Omega)\right)$. Besides, combinig (42) and (41) gives $\nabla_{x} S_{\psi}(0)=m \frac{J(0)}{n(0)}$. To check that Eq. (26) holds strongly, we first fix $t \in[0, T)$. For $t>0$, we can differentiate $S_{\psi}$ to get

$$
\nabla_{x} S_{\psi}(t)=\nabla_{x} S_{0}(t)-\nabla_{x}\left(\int_{0}^{t} K[n, J](s, x) d s\right) .
$$

In virtue of Schwartz's lemma and Lemma 3.3 (iii) we obtain

$$
\nabla_{x} S_{\psi}(t)=\nabla S_{0}(t)-m \int_{0}^{t} \partial_{t}\left(\frac{J}{n}\right)(s) d s,
$$

which implies $\nabla_{x} S_{\psi}(t)=m(J / n)(t)$. Then, Lemma 3.3 ensures that $\nabla_{x} S_{\psi} \in$ $C\left([0, T), H^{1}(\Omega)\right)$, so that $S_{\psi}$ is easily seen to belong to $C\left((0, T) ; H^{2}(\Omega)\right)$. Since $\int_{\Omega} S_{\psi} d x=\int_{\Omega} S_{0} d x$ for all $t \in[0, T)$, Lemma 3.1 (ii) implies that $S_{\psi} \in$ $C\left([0, T) ; H^{2}(\Omega)\right)$ and that Eq. (26) holds strongly for all $t \in[0, T)$. A simple exercise involving the facts that $\partial_{t} S_{\psi} \in C\left((0, T) ; L^{2}(\Omega)\right)$ and $S_{\psi}$ is continuous at $t=0$ then leads to $S_{\psi} \in X^{T}$. Now, from the definition and regularity of $K$ it is a simple matter to deduce that Eq. (40) is fulfilled in a strong sense. As consequence, $S_{\psi}$ defined by (42) satisfies (i) and (ii).

To prove that (iii) is also satisfied we define

$$
\psi^{S}(t, x)=\sqrt{n(t, x)} \exp \left\{\frac{i}{\alpha} S_{\psi}(t, x)\right\} \quad \forall t \in[0, T) \text {, a.e. } x \in \Omega .
$$


It is straightforward to check that $\psi^{S} \in X_{\delta}^{T}$. Furthermore, the computation of $\nabla\left(\psi / \psi^{S}\right)$ leads us to the relation $\psi(t, x)=z(t) \psi^{S}(t, x)$ for certain $z \in$ $C^{1}\left([0, T) ; L^{2}(\Omega)\right)$ verifying

$$
z^{\prime}=\frac{1}{\left(\psi^{S}\right)^{2}}\left(\psi^{S} \partial_{t} \psi-\psi \partial_{t} \psi^{S}\right)
$$

We do know by hypothesis (cf. (38)) that $\partial_{t} \psi=\frac{i \alpha}{2 m} \Delta_{x} \psi-i \Theta[n, J] \psi$. Then, invoking Lemma 3.2 (ii) we have that

$$
\partial_{t} \psi=\left(-\frac{i \alpha}{\hbar^{2}} Q-\frac{m i}{2 \alpha} \frac{|J|^{2}}{n^{2}}-\frac{\nabla_{x} \cdot J}{2 n}-i \Theta[n, J]\right) \psi
$$

On the other hand $\partial_{t} \psi^{S}=\left(\frac{1}{2 n} \partial_{t} n+\frac{i}{\alpha} \partial_{t} S_{\psi}\right) \psi^{S}$, and an application of Lemmata 3.2 (i) and 3.3 (iii) gives

$$
\begin{array}{r}
\partial_{t} \psi^{S}=\left\{-\frac{\nabla_{x} \cdot J}{2 n}+\operatorname{Im}(\Theta[n, J])+\frac{i}{\alpha}\left(-\frac{\alpha^{2}}{\hbar^{2}} Q-\frac{m}{2} \frac{|J|^{2}}{n^{2}}-\alpha \operatorname{Re}(\Theta[n, J])\right)\right\} \psi^{S} \\
\left.=\left\{-\frac{i \alpha}{\hbar^{2}} Q-\frac{m i}{2 \alpha} \frac{|J|^{2}}{n^{2}}-\frac{\nabla_{x} \cdot J}{2 n}-i \Theta[n, J]\right)\right\} \psi^{S},
\end{array}
$$

so that $z^{\prime}=0$ and thus $z \equiv z_{0} \in \mathbb{C}$. As consequence we may write

$$
\psi(t, x)=z_{0} \sqrt{n(t, x)} \exp \left\{\frac{i}{\alpha} S_{\psi}(t, x)\right\} \quad \forall t \in[0, T), \text { a.e. } x \in \Omega \text {. }
$$

In particular, if $t=0$ Eq. (41) allows to get $z_{0}=1$. Therefore, $S_{\psi}$ satisfies the Madelung decomposition established in (10). Furthermore, by defining $S_{\psi}^{l}(t, x)=$ $S_{\psi}(t, x)+2 \pi \alpha l$ for $t \in[0, T)$, a.e. $\Omega$ and $l \in \mathbb{Z}$, we find that $\left\{S_{\psi}^{l}\right\}_{l \in \mathbb{Z}} \subset X^{T}$ fulfills (iii).

To end up, given $S \in X^{T}$ satisfying (ii) it is a simple matter to check from Eq. (26) that $\nabla_{x} S=\nabla_{x} S_{\psi}$, hence $S(t, x)=S_{\psi}(t, x)+l(t)$ with $l \in C^{1}([0, T))$. Now, making use of (ii) we have that $S(t, x)=S_{\psi}(t, x)+l_{0}$ with $l_{0}$ constant. Actually, Eq. (39) implies that $l_{0} \in 2 \pi \alpha \mathbb{Z}$ in such a way that $S \in\left\{S_{\psi}^{l}\right\}$. This shows (iv).

Now we are done with the proof.

Remark 3.2. Notice that prescribing the value $\int_{\Omega} S d x=\mu \in \mathbb{R}$ allows us to obtain an unique, regular $S(t, x)$ (both in time and space) which satisfies the Madelung decomposition (10), that is, any solution of a Schrödinger equation has an argument function with constant-in-time mean value. 


\section{On the Equivalence Between the Full Logarithmic and the Purely Logarithmic Schrödinger Equations}

Once the existence of a regular argument function has been established, the transformation $F$ introduced in (11) as well as its inverse operator

$$
F^{-1}(\phi)(t, x)=\phi(t, x) \exp \left\{i \log \left(\sqrt{n_{\phi}(t, x)}\right)\right\}
$$

make full sense and the equivalence between both Full Logarithmic and Purely Logarithmic initial-boundary value problems can be tackled. In the following we shall take advantage from the fact that $F$ (as well as $F^{-1}$ ) preserves the amplitude of the wavefunction and use $n=|\psi|^{2}=|\phi|^{2}$ indistinctively. Firstly we set up the abstract frame in which $F$ keeps the $H_{\delta}^{2}$ regularity in a one-to-one way. As a matter of fact we prove that $F$ is homeomorphic on $C\left([0, T) ; H_{\delta}^{2}\right)$, which in particular guarantees the preservation of the dynamical behaviours.

Proposition 4.1. Let $\Omega \subset \mathbb{R}^{3}$ be a bounded domain and $\delta>0$. Given $\psi \in$ $C\left([0, T) ; H_{\delta}^{2}\right)$, consider $F(\psi)$ defined as in (11). Then, $F: C\left([0, T) ; H_{\delta}^{2}\right) \rightarrow$ $C\left([0, T) ; H_{\delta}^{2}\right)$ is an homeomorphism (for the $H^{2}$ norm) with inverse operator $F^{-1}$, defined as stated in (44).

Proof. Given $\mathcal{F}: X \rightarrow X$ an homeomorphism and defining $F(x)(t):=\mathcal{F}(x(t))$ for all $x \in C([0, T) ; X)$ and $t \in[0, T)$, it is clear that $F: C([0, T) ; X) \rightarrow C([0, T) ; X)$ is also an homeomorphism. Then, it is enough to show that $\mathcal{F}: H_{\delta}^{2} \rightarrow H_{\delta}^{2}$ defines an homeomorphic operator. We split the proof into two steps.

Step 1: $\mathcal{F}\left(H_{\delta}^{2}\right) \subseteq H_{\delta}^{2}$. Given $\psi \in H_{\delta}^{2}$ we have $n_{\mathcal{F}(\psi)}=n_{\psi}$, hence $\mathcal{F}(\psi) \in L^{2}(\Omega)$ and $|\mathcal{F}(\psi)|>\delta$ a.e. $\Omega$. Differentiating now $\mathcal{F}(\psi)$ we find

$$
\nabla_{x} \mathcal{F}(\psi)=\left(\nabla_{x} \psi-i \frac{\nabla_{x} n}{2 n} \psi\right) \exp \{-i \log (\sqrt{n})\}
$$

Then, in virtue of Lemma 3.2 we easily deduce that $\nabla_{x} \mathcal{F}(\psi) \in\left(L^{2}(\Omega)\right)^{3}$. In addition

$$
\begin{aligned}
\nabla_{x} \otimes \nabla_{x} \mathcal{F}(\psi)=\left\{\nabla_{x} \otimes \nabla_{x} \psi-\right. & \frac{i}{2}\left(\frac{\nabla_{x} \otimes \nabla_{x} n}{n}\right) \psi+\frac{2 i-1}{4}\left(\frac{\nabla_{x} n}{n} \otimes \frac{\nabla_{x} n}{n}\right) \psi \\
& \left.-\frac{i}{n} \operatorname{Sym}\left(\nabla_{x} n \otimes \nabla_{x} \psi\right)\right\} \exp \{-i \log (\sqrt{n})\},
\end{aligned}
$$

where we denoted $\operatorname{Sym}\left(\nabla_{x} n \otimes \nabla_{x} \psi\right)$ the symmetric part of the rank-two tensor $\nabla_{x} n \otimes \nabla_{x} \psi$. Since $\psi \in H_{\delta}^{2}$, we have $\nabla_{x} \otimes \nabla_{x} \psi \in\left(L^{2}(\Omega)\right)^{9}$. Likewise, the fact that $n \in H_{\delta^{2}}^{2}$ yields $\left(\nabla_{x} \otimes \nabla_{x} n\right) \psi / n \in\left(L^{2}(\Omega)\right)^{9}$. Besides

$$
\nabla_{x} \psi, \frac{\nabla_{x} n}{n} \in\left(H^{1}(\Omega)\right)^{3} \subset L^{4}(\Omega)
$$


which implies $\left(\nabla_{x} n \otimes \nabla_{x} n\right) \psi / n^{2}, \nabla_{x} \psi \otimes \nabla_{x} \psi,\left(\nabla_{x} n / n\right) \otimes \nabla_{x} \psi \in\left(L^{2}(\Omega)\right)^{3}$, so that $\mathcal{F}(\psi)$ is readily seen to belong to $H_{\delta}^{2}$.

Step 2: $\mathcal{F}$ is one-to-one and bicontinuous. Formulae (11) and (44) make it clear that $\mathcal{F}\left(\mathcal{F}^{-1}(\phi)\right)=\phi$ and $\mathcal{F}^{-1}(\mathcal{F}(\psi))=\psi$ for all $\phi, \psi \in H_{\delta}^{2}$. As consequence, $\mathcal{F}$ is clearly one-to-one. To analyze bicontinuity it is enough to show that $\mathcal{F}$ is continuous, as for $\mathcal{F}^{-1}$ the argument is analogous.

Let $\psi \in H_{\delta}^{2}$ and $\left\{\psi_{k}\right\} \subset H_{\delta}^{2}$ such that $\psi_{k} \rightarrow \psi$ in the $H^{2}$ norm. Since $\Omega$ is bounded, we can estimate

$$
\begin{aligned}
\left\|\mathcal{F}\left(\psi_{k}\right)-\mathcal{F}(\psi)\right\|_{L^{2}} \leq & \sqrt{|\Omega|}\left\{\left\|\left(\psi_{k}-\psi\right) \exp \{-i \log (\sqrt{n})\}\right\|_{L^{\infty}}\right. \\
& \left.+\left\|\psi_{k}\left(\exp \left\{-i \log \left(\sqrt{n_{k}}\right)\right\}-\exp \{-i \log (\sqrt{n})\}\right)\right\|_{L^{\infty}}\right\} .
\end{aligned}
$$

The first term in the right-hand side can be straightforwardly bounded by $\| \psi_{k}-$ $\psi \|_{L^{\infty}}$. For the second term, Rellich-Kondrachov's compactness theorem along with the convergence $n_{k} \rightarrow n$ in $H^{2}(\Omega)$ (cf. Lemma 3.2) and the fact that $n>\delta^{2}$, imply that $\log \left(\sqrt{n_{k}}\right) \rightarrow \log (\sqrt{n})$ in $L^{\infty}(\Omega)$. Consequently

$$
\begin{aligned}
& \| \psi_{k}(\exp \{\left.\left.-i \log \left(\sqrt{n_{k}}\right)\right\}-\exp \{-i \log (\sqrt{n})\}\right) \|_{L^{\infty}} \\
& \leq C\left\|\psi_{k}\right\|_{H^{2}}\left\|\log \left(\sqrt{n_{k}}\right)-\log (\sqrt{n})\right\|_{L^{\infty}} \rightarrow 0 .
\end{aligned}
$$

Now, employing the identities (45) and (46) and the continuity of the derivatives of $n$ and $\psi$ from $H_{\delta}^{2}$ onto $L^{2}(\Omega)$, it is a simple task to prove that $\mathcal{F}\left(\psi_{k}\right) \rightarrow \mathcal{F}(\psi)$ in $H^{2}(\Omega)$. This ends the proof.

Remark 4.1. In the sequel we shall make use of Proposition 4.1 to give sense to the action of $F$ on the boundary condition $\psi_{B} \in H^{3 / 2}(\partial \Omega)$. To this aim we just note that, for functions in $H_{\delta}^{2}$, formulae (11) and (44) are valid pointwise in $\Omega$, thus in $\partial \Omega$. Then, defining $F\left(\psi_{B}\right):=F\left(\psi_{0}\right)_{\mid \partial \Omega}$ we have that $F\left(\psi_{B}\right) \in H^{3 / 2}(\partial \Omega)$ and that the identities

$$
F\left(F^{-1}\left(\phi_{B}\right)\right)=\phi_{B}, F^{-1}\left(F\left(\psi_{B}\right)\right)=\psi_{B} \quad \forall x \in \partial \Omega, \forall \phi_{B}, \psi_{B} \in H^{3 / 2}(\partial \Omega)
$$

hold.

Our next result shows how the solutions to both problems are linked each other through $F$. The idea is the following: (i) Given a solution of the initialboundary value problem for the PLSE (respectively, the FLSE), Theorem 3.2 applies to get the corresponding hydrodynamic system. (ii) This potentialflow system is linearly connected to its FLSE (respectively, PLSE) counterpart (cf. Corollary 4.1). (iii) The solutions to the latter provide the ingredients for the effective reconstruction of a solution to the initial-boundary value problem for the FLSE (respectively, the PLSE), that will be shown a posteriori to be unique (cf. Theorem 4.1 (iii)).

Theorem 4.1. Let $T>0$ and $\Omega \subset \mathbb{R}^{3}$ satisfying (H1). Then, the following assertions hold true. 
(i) Let $\psi_{0}, \psi_{B}$ satisfying (H2) and (H3) be such that

$$
F\left(\psi_{0}\right)=: \phi_{0} \in H_{\delta}^{2}, \quad F\left(\psi_{B}\right)=: \phi_{B} \in H_{\delta}^{3 / 2}(\partial \Omega) .
$$

Let also $\phi \in X_{\delta}^{T}$ be a strong solution of $(12)-(14)$ in $[0, T)$. Then, $\psi=$ $F^{-1}(\phi)$ is a strong solution of $(3)-(6)$ in $[0, T)$.

(ii) Conversely, let $\phi_{0}, \phi_{B}$ satisfying $(\mathrm{H} 2)$ and (H3) be such that

$$
F^{-1}\left(\phi_{0}\right)=: \psi_{0} \in H_{\delta}^{2}, \quad F^{-1}\left(\phi_{B}\right)=: \psi_{B} \in H_{\delta}^{3 / 2}(\partial \Omega) .
$$

Let also $\psi \in X_{\delta}^{T}$ be a strong solution of (3)-(6) in $[0, T)$. Then, $\phi=F(\psi)$ is a strong solution of $(12)-(14)$ in $[0, T)$.

(iii) Uniqueness is inheritable via $F$, namely if $\psi_{0}, \psi_{B}$ satisfy $(\mathrm{H} 2)$ and $(\mathrm{H} 3)$ and the problem (12)-(14) admits a unique solution, then (3)-(6) also admits a unique solution. Likewise, if $\phi_{0}, \phi_{B}$ satisfy $(\mathrm{H} 2)$ and $(\mathrm{H} 3)$ and the problem (3)-(6) admits a unique solution, then (12)-(14) also admits a unique solution.

Proof. Let $\phi \in X_{\delta}^{T}$ be a strong solution of (12)-(14) satisfying the hypotheses of Theorem 3.2 with $\Theta[n]=\frac{\Lambda}{\alpha} \log (n)$. Then, a function $S_{\phi} \in X^{T}$ does exist that verifies

$$
\phi(t, x)=\sqrt{n(t, x)} \exp \left\{\frac{i}{\alpha} S_{\phi}(t, x)\right\} .
$$

In particular, Eqs. (26) and (40) are fulfilled, which along with Lemma 3.3 allow to conclude that the couple $\left(n, S_{\phi}\right)$ solves the following hydrodynamic system strongly in $[0, T)$

$$
\begin{aligned}
\partial_{t} n+\frac{1}{m} \nabla_{x} \cdot\left(n \nabla_{x} S_{\phi}\right) & =0, \\
\partial_{t} S_{\phi}+\frac{1}{2 m}\left|\nabla_{x} S_{\phi}\right|^{2} & =-\frac{\alpha^{2}}{\hbar^{2}} Q-\Lambda \log (n) .
\end{aligned}
$$

Defining now

$$
S_{\psi}(t, x):=S_{\phi}(t, x)+\alpha \log (\sqrt{n(t, x)}),
$$

the following system is satisfied strongly by $\left(n, S_{\psi}\right)$ after simple calculations

$$
\begin{aligned}
\partial_{t} n+\frac{1}{m} \nabla_{x} \cdot\left(n \nabla_{x} S_{\psi}\right) & =D_{q q} \Delta_{x} n, \\
\partial_{t} S_{\psi}+\frac{1}{2 m}\left|\nabla_{x} S_{\psi}\right|^{2} & =-\frac{2 \alpha^{2}}{\hbar^{2}} Q-\Lambda \log (n)-D_{q q} \Delta_{x} S_{\psi} .
\end{aligned}
$$

If we now define $\psi:=F^{-1}(\phi)$ we have

$$
\psi(t, x)=\sqrt{n(t, x)} \exp \left\{\frac{i}{\alpha} S_{\psi}(t, x)\right\},
$$


so that the evolution of $\psi$ can be easily computed in terms of the following Schrödinger equation

$i \alpha \partial_{t} \psi=-\frac{\alpha^{2}}{2 m} \Delta_{x} \psi+\frac{\alpha^{2}}{\hbar^{2}} Q \psi+\Lambda \log (n) \psi+\frac{i \alpha D_{q q}}{2}\left(\frac{\Delta_{x} n}{n}\right) \psi+m D_{q q} \nabla_{x} \cdot\left(\frac{J}{n}\right) \psi$

in a strong sense, thus $\psi$ is a strong solution of $(3)-(4)$ in $[0, T)$. Furthermore, the following identities hold almost everywhere in $\Omega$ and $\partial \Omega$, respectively:

$$
\begin{aligned}
\psi(0, x) & =\sqrt{n_{0}(x)} \exp \left\{\frac{i}{\alpha}\left(S_{\phi}(0, x)+\alpha \log \left(\sqrt{n_{0}(x)}\right)\right)\right\} \\
& =\phi_{0}(x) \exp \left\{i \log \left(\sqrt{n_{0}(x)}\right)\right\}=\psi_{0}(x), \\
\psi(t, x) & =\sqrt{n(t, x)} \exp \left\{\frac{i}{\alpha}\left(S_{\phi}(t, x)+\alpha \log (\sqrt{n(t, x)})\right)\right\} \\
& =\phi_{B}(x) \exp \left\{i \log \left(\sqrt{n_{B}(x)}\right)\right\}=\psi_{B}(x) .
\end{aligned}
$$

Consequently, $\psi$ solves (3)-(6). This proves (i).

The proof of (ii) is fully analogous to that of (i), by just taking into account that now

$$
\Theta[n, J]=\frac{\alpha}{\hbar^{2}} Q+\frac{\Lambda}{\alpha} \log (n)+\frac{i D_{q q}}{2}\left(\frac{\Delta_{x} n}{n}\right)+\frac{1}{2} \nabla_{x} \cdot\left(\frac{J}{n}\right)
$$

is a continuous operator from $H_{\delta^{2}}^{2} \times\left(H^{1}(\Omega)\right)^{3}$ onto $L^{2}(\Omega)$, and the fact that

$$
S_{\phi}(t, x)=S_{\psi}(t, x)-\alpha \log (\sqrt{n(t, x)}) .
$$

To prove (iii) we firstly assume that $\psi_{1}, \psi_{2} \in X_{\delta}^{T}$ are two strong solutions of (3)-(6). It is clear that $\phi_{0}(x)=\psi_{0}(x) \exp \left\{-i \log \left(\sqrt{n_{0}(x)}\right)\right\}$ and $\phi_{B}(x)=$ $\psi_{B}(x) \exp \left\{-i \log \left(\sqrt{n_{B}(x)}\right)\right\}$ satisfy (H2) and (H3). Besides, $\psi_{1,2}(0, x)=$ $F^{-1}\left(\phi_{0}\right)(x)$ and $\psi_{1,2}(t, x)=F^{-1}\left(\phi_{B}\right)(t, x)$ for all $t \in[0, T)$, almost everywhere in $\partial \Omega$. As consequence, $\psi_{1,2}$ fulfill the assumptions required in (ii) so that

$$
\phi_{1,2}(t, x)=F\left(\psi_{1,2}\right)(t, x)=\psi_{1,2}(t, x) \exp \left\{-i \log \left(\sqrt{n_{1,2}(t, x)}\right)\right\}
$$

belong to $X_{\delta}^{T}$ and are strong solutions of (12)-(14). As this problem is assumed to enjoy uniqueness, there should be $\phi_{1}=\phi_{2}$, hence

$$
\psi_{1} \exp \left\{-i \log \left(\sqrt{n_{1}}\right)\right\}=\psi_{2} \exp \left\{-i \log \left(\sqrt{n_{2}}\right)\right\}, \quad n_{1,2}=\left|\phi_{1,2}\right|^{2},
$$

from which we straightforwardly deduce that $n_{1}=n_{2}$, therefore $\psi_{1}=\psi_{2}$. The reciprocal assertion is analogous.

It is noticeable the fact that we have established the equivalence between Eqs. (3) and (12) via the systems (50)-(51) and (47)-(48), respectively. More precisely, the proof of Theorem 4.1 requires both systems to be biunivoquely linked, 
which is true because of the linearity of the correspondence $\left(n, S_{\psi}\right) \leftrightarrow\left(n, S_{\phi}\right)$. Then, we might end this section with the following

Corollary 4.1. Assume that (H1) is fulfilled and let $T>0$. Then

(i) If $\left(n, S_{\phi}\right) \in X_{\delta}^{T} \times X^{T}$ is a strong solution of the system (47)-(48) in $[0, T)$, then $\left(n, S_{\psi}\right) \in X_{\delta}^{T} \times X^{T}$ is a strong solution of the system (50)-(51) in $[0, T)$, where $S_{\psi}$ is that given in (49).

(ii) Conversely, if $\left(n, S_{\psi}\right) \in X_{\delta}^{T} \times X^{T}$ is a strong solution of the system (50)(51) in $[0, T)$, then $\left(n, S_{\phi}\right)$ is a strong solution of the system (47)-(48) in $[0, T)$, where $S_{\phi}$ is that given in $(52)$.

\section{An auxiliary Problem: Local Wellposedness of the Purely LOGARITHMIC SCHRÖDINGER EQUATION}

In this section we shall handle the auxiliary mixed initial-boundary value problem for the PLSE (12) in $H_{\delta}^{2}$. Indeed, our equation of interest (3) has been shown to be equivalent to Eq. (12) by means of a suitable nonlinear gauge transformation $F$ (cf. (11)), in such a manner that strong solutions of Eq. (12) are straightforwardly led to strong solutions of Eq. (3) via the action of $F$ (cf. Theorem 4.1).

Wellposedness of Eq. (12) has already been dealt with in the literature [12, 14], mainly for negative values of $\Lambda$ (in which case stable Gaussons do exist [13]) or in the whole space case. In particular, the multidimensional whole space problem for both signs of the logarithmic nonlinearity has been recently investigated by some of the authors in [29]. Eq. (12) fits Davidson's sign choice [19]. The single sign choice for the logarithmic term first made in [9] and later continued in $[13,14]$ was owing to the fact that the other sign typically leads to an energy functional which is not bounded from below. However, the positive sign for the logarithmic nonlinearity was physically justified in [19] as representing a diffusion force within the context of stochastic quantum mechanics and analyzed in [41] in the context of the Schrödinger-Langevin-Kostin equation accounting for negative dissipation.

Here, we are concerned with an $H^{2}$ theory for the problem consisting of solving

$$
i \alpha \partial_{t} \phi=-\frac{\alpha^{2}}{2 m} \Delta_{x} \phi+\Lambda \log (n) \phi, \quad n=|\phi|^{2},
$$

in a nonvacuum bounded region $\Omega$ along with the compatible initial-boundary conditions

$$
\phi(0, x)=\phi_{0}(x) \text { in } \Omega, \quad \phi(t, x)=\phi_{B}(x) \text { in }[0, T) \times \partial \Omega .
$$

Our main result in this section is the following.

Theorem 5.1. Let $\Omega \subset \mathbb{R}^{3}$ be a $C^{2}$ bounded domain. Let also $\phi_{0} \in H^{2}(\Omega)$ and $\phi_{B} \in H^{3 / 2}(\partial \Omega)$ satisfy $(\mathrm{H} 2)-(\mathrm{H} 3)$. Then, there exists $T=T\left(\delta, \phi_{0}, \phi_{B}, \Omega\right)>0$ and a unique strong solution $\phi \in X_{\delta}^{T}$ of Eq. (53) in $[0, T)$ satisfying the initial/boundary conditions stated in (54). 
For the sake of clarity, first of all we define the concept of mild solution for this problem.

Definition 5.1. Given $T>0$, we will say that $\phi \in C\left([0, T) ; H^{2}(\Omega)\right)$ is a mild solution of the logarithmic Schrödinger equation (53) subject to the initial-boundary conditions (54) if it solves the following integral equation

$$
\phi(t, x)=U(t)+V(t)-\frac{i \Lambda}{\alpha} \int_{0}^{t} e^{A(t-s)}\left[\log (n(s, x)) \phi(s, x)-G_{B}\right] d s,
$$

where $A=i(\alpha / 2) \Delta_{x}$ is the generator of the evolution semigroup with domain

$$
D=H_{0}^{1}(\Omega) \cap H^{2}(\Omega),
$$

and where $U$ and $V$ respectively solve the following linear Schrödinger problems

$$
\begin{aligned}
& U \in X^{T}, \\
& i \partial_{t} U=-D_{q q} \Delta_{x} U \quad \text { in }[0, T) \times \Omega, \\
& U(0, x)=\phi_{0}(x) \text { in } \Omega, \\
& U(t, x)=\phi_{B}(x) \text { in } \partial \Omega, \quad t \in[0, T),
\end{aligned}
$$

and

$$
\begin{aligned}
& V \in C([0, T) ; D) \cap C^{1}\left([0, T) ; L^{2}(\Omega)\right), \\
& i \partial_{t} V=-D_{q q} \Delta_{x} V+\frac{\Lambda}{\alpha} G_{B} \quad \text { in }[0, T) \times \Omega, \\
& V(0, x)=0 \quad \text { in } \Omega, \\
& V(t, x)=0 \quad \text { in } \partial \Omega, \quad t \in[0, T),
\end{aligned}
$$

$G_{B}$ being the harmonic extension to $\Omega$ of $\log \left(\left|\phi_{B}\right|^{2}\right) \phi_{B}$.

Remark 5.1. Notice that the Rellich-Kondrachov compactness theorem along with (H3) guarantee the existence of a continuous representative of $\log \left(\left|\phi_{B}\right|^{2}\right) \phi_{B}$ along $\partial \Omega$. Since $\Omega$ is $C^{2}$ by hypothesis, it can be claimed that there exists $G_{B} \in$ $C^{2}(\Omega) \cap C(\bar{\Omega})$ harmonic in $\Omega$ and such that $G_{B}=\log \left(\left|\phi_{B}\right|^{2}\right) \phi_{B}$ in $\partial \Omega$, i.e. the harmonic extension to $\Omega$ of $\log \left(\left|\phi_{B}\right|^{2}\right) \phi_{B}$.

To prove the existence of mild solutions to Eq. (53) subject to (54) we will use the Banach fixed-point theorem applied to the operator

$$
\Gamma(\phi):=U(t)+V(t)-\frac{i \Lambda}{\alpha} \int_{0}^{t} e^{A(t-s)}\left[\log (n(s)) \phi(s)-G_{B}\right] d s,
$$

defined on an adequate subset of $X_{\delta}^{T}$. Clearly, any fixed point $\phi \in X_{\delta}^{T}$ of $\Gamma$ is a mild solution of (53) with (54). We start by showing that the nonlinear potential $\log (n)$ is contractive and then establish an appropriate subset for which the operator $\Gamma$ is closed, in order to meet the hypotheses of Banach's fixed point theorem. Finally, we prove that our mild solution is actually a strong solution. 
Lemma 5.1. Under the hypotheses of Theorem 5.1, there exist positive constants $r_{0}, T_{0}, K_{0}$, depending upon $\delta, \Omega, \phi_{0}$ and $\phi_{B}$, such that

$$
\|U\|_{L^{\infty}\left([0, T) ; H^{2}(\Omega)\right)},\|V\|_{L^{\infty}\left([0, T) ; H^{2}(\Omega)\right)} \leq K_{0},
$$

and $B_{r, T} \subset C\left([0, T) ; H_{\delta}^{2}\right)$ for all $r \in\left(0, r_{0}\right)$ and $T \in\left(0, T_{0}\right)$, where

$$
B_{r, T}:=\left\{\phi \in C\left([0, T) ; H^{2}(\Omega)\right):\|\phi-U-V\|_{L^{\infty}\left([0, T) ; H^{2}(\Omega)\right)} \leq r\right\} .
$$

Proof. Let

$$
\begin{aligned}
m_{0} & =\operatorname{ess}-\inf \left\{\left|\phi_{0}(x)\right|: x \in \Omega\right\}, \quad m_{B}=\operatorname{ess-inf}\left\{\left|\phi_{B}(x)\right|: x \in \partial \Omega\right\}, \\
m & =\min \left\{m_{0}, m_{B}\right\} .
\end{aligned}
$$

By (H2) and (H3) (cf. Remark 5.1) we have $m>\delta$, so that there exists $\varepsilon>0$ such that $\varepsilon<m-\delta$. Define $r_{0}:=\frac{\varepsilon}{3 C_{1}}$, with $C_{1}$ as in the proof of Lemma 5.2 The continuity in time of $U$ and $V$ guarantees the existence of $T_{0}>0$ such that

$$
\left\|U(t)-\phi_{0}\right\|_{H^{2}},\|V(t)\|_{H^{2}}<r_{0} \quad \forall 0 \leq t<T_{0} .
$$

We also define $K_{0}=r_{0}+\left\|\phi_{0}\right\|_{H^{2}}$ and consider $0<r<r_{0}$ and $0<T<T_{0}$. Then, the first claim is obvious according to (58). To check the second one, let $\phi \in B_{r, T}$. We have

$$
\begin{aligned}
\|\phi(t)-\phi(0)\|_{H^{2}} & \leq\|\phi(t)-U(t)-V(t)\|_{H^{2}}+\left\|U(t)-\phi_{0}\right\|_{H^{2}}+\|V(t)\|_{H^{2}} \\
& <3 r_{0}=\frac{\varepsilon}{C_{1}}
\end{aligned}
$$

for all $t \in[0, T)$, thus $\left\|\phi-\phi_{0}\right\|_{L^{\infty}\left([0, T) ; H^{2}(\Omega)\right)}<\frac{\varepsilon}{C_{1}}$. Also, the Sobolev embedding $H^{2}(\Omega) \hookrightarrow L^{\infty}(\Omega)$ allows to claim that $\left|\phi_{0}(x)\right|-|\phi(t, x)| \leq\left|\phi_{0}(x)-\phi(t, x)\right|<\varepsilon$ a.e. $x \in \Omega$ for all $t \in[0, T)$, hence $|\phi(t, x)|>\left|\phi_{0}(x)\right|-\varepsilon>m_{0}-m+\delta \geq \delta$ and $\phi \in C\left([0, T) ; H_{\delta}^{2}\right)$. The arbitrariness of $\phi$ ends the proof.

Lemma 5.2 (a priori estimates). Let $M, \delta>0$ and $\Omega \subset \mathbb{R}^{3}$ bounded. Then the following assertions hold true.

(i) There exists $K_{1}(\delta, M, \Omega)>0$ such that $\|\phi \log (n)\|_{H^{2}} \leq K_{1}$ for all $\phi \in H_{\delta}^{2}$ such that $\|\phi\|_{H_{2}} \leq M$.

(ii) There exists $K_{2}(\delta, M, \Omega)>0$ such that

$$
\left\|\phi_{1} \log \left(n_{1}\right)-\phi_{2} \log \left(n_{2}\right)\right\|_{L^{2}} \leq K_{2}\left\|\phi_{1}-\phi_{2}\right\|_{L^{2}}
$$

for all $\phi_{1}, \phi_{2} \in H_{\delta}^{2}$ such that $\left\|\phi_{1,2}\right\|_{H_{2}} \leq M$.

(iii) There exists $K_{3}(\delta, M, \Omega)>0$ such that

$$
\left\|\phi_{1} \log \left(n_{1}\right)-\phi_{2} \log \left(n_{2}\right)\right\|_{H^{2}} \leq K_{3}\left\|\phi_{1}-\phi_{2}\right\|_{H^{2}}
$$

for all $\phi_{1}, \phi_{2} \in H_{\delta}^{2}$ such that $\left\|\phi_{1,2}\right\|_{H_{2}} \leq M$. 
Proof. Consider constants $C_{1}, C_{2}>0$ such that $\|\phi\|_{L^{\infty}} \leq C_{1}\|\phi\|_{H^{2}} \leq C_{1} M$ and $\|\phi\|_{L^{4}} \leq C_{2}\|\phi\|_{H^{1}} \leq C_{2} M$ for any $\phi \in C_{c}^{\infty}(\Omega)$. Define also

$$
M_{1}=\max \left\{|\log (\delta)|, 2\left|\log \left(C_{1} M\right)\right|\right\} .
$$

(i) The $L^{2}$ estimate straightforwardly yields

$$
\|\phi \log (n)\|_{L^{2}} \leq M_{1} M .
$$

Estimating the first order derivative is also a simple matter

$$
\left\|\nabla_{x}(\phi \log (n))\right\|_{L^{2}} \leq M_{1} M+\frac{C_{1}}{\delta^{2}} M\|n\|_{H^{2}} .
$$

Finally, taking into account that

$$
\begin{aligned}
\nabla_{x} \otimes \nabla_{x}(\phi \log (n))= & \left(\nabla_{x} \otimes \nabla_{x} \phi\right) \log (n)+2 \operatorname{Sym}\left(\nabla_{x} \phi \otimes \frac{\nabla_{x} n}{n}\right) \\
& +\phi\left(\frac{\nabla_{x} \otimes \nabla_{x} n}{n}-\frac{\nabla_{x} n}{n} \otimes \frac{\nabla_{x} n}{n}\right)
\end{aligned}
$$

we find

$$
\begin{aligned}
\left\|\nabla_{x} \otimes \nabla_{x}(\phi \log (n))\right\|_{L^{2}} \leq & M_{1} M+\frac{2 C_{2}^{2}}{\delta^{2}} M\|n\|_{H^{2}} \\
& +\frac{C_{1}}{\delta^{2}} M\|n\|_{H^{2}}\left(1+\frac{C_{2}^{2}}{\delta^{2}}\|n\|_{H^{2}}\right) .
\end{aligned}
$$

Combining (59), (60) and (62) and noticing that

$$
\|n\|_{H^{2}} \leq\left(5 C_{1}+2 C_{2}^{2}\right) M^{2}
$$

we are done with the proof.

(ii) We have

$$
\begin{aligned}
\left\|\phi_{1} \log \left(n_{1}\right)-\phi_{2} \log \left(n_{2}\right)\right\|_{L^{2}} \leq & \left\|\phi_{1}\left(\log \left(n_{1}\right)-\log \left(n_{2}\right)\right)\right\|_{L^{2}} \\
& +\left\|\log \left(n_{2}\right)\left(\phi_{1}-\phi_{2}\right)\right\|_{L^{2}} \\
\leq & \left(\frac{2 C_{1}}{\delta} M+M_{1}\right)\left\|\phi_{1}-\phi_{2}\right\|_{L^{2}},
\end{aligned}
$$

for which we used the fact that $\left|\log \left(r_{1}^{2}\right)-\log \left(r_{2}^{2}\right)\right| \leq \frac{2}{\delta}\left|r_{1}-r_{2}\right|$ as directly sheds from the mean value theorem. This ends this part of the proof.

(iii) We first compute

$$
\begin{aligned}
\nabla_{x}\left[\phi_{1}\left(\log \left(n_{1}\right)-\log \left(n_{2}\right)\right)\right]= & \nabla_{x} \phi_{1}\left(\log \left(n_{1}\right)-\log \left(n_{2}\right)\right) \\
& +\phi_{1}\left[\frac{\nabla_{x}\left(n_{1}-n_{2}\right)}{n_{1}}+\frac{\nabla_{x} n_{2}}{n_{1} n_{2}}\left(n_{2}-n_{1}\right)\right]
\end{aligned}
$$


and estimate

$$
\begin{gathered}
\left\|\nabla_{x} \phi_{1}\left(\log \left(n_{1}\right)-\log \left(n_{2}\right)\right)\right\|_{L^{2}} \\
\leq \frac{2}{\delta}\left\|\nabla_{x} \phi_{1}\left(\left|\phi_{1}\right|-\left|\phi_{2}\right|\right)\right\|_{L^{2}} \\
\leq \frac{2 C_{2}^{2}}{\delta} M\left\|\phi_{1}-\phi_{2}\right\|_{H^{1}}, \\
\left\|\frac{\nabla_{x}\left(n_{1}-n_{2}\right)}{n_{1}}\right\|_{L^{2}} \leq \frac{2}{\delta^{2}}\left\|\overline{\phi_{1}}\left(\nabla_{x} \phi_{1}-\nabla_{x} \phi_{2}\right)-\left(\overline{\phi_{2}}-\overline{\phi_{1}}\right) \nabla_{x} \phi_{2}\right\|_{L^{2}} \\
\leq \frac{4 C_{1}}{\delta^{2}} M\left\|\phi_{1}-\phi_{2}\right\|_{H^{2}}, \\
\left\|\frac{\nabla_{x} n_{2}}{n_{1} n_{2}}\left(n_{2}-n_{1}\right)\right\|_{L^{2}} \leq \frac{2}{\delta^{4}}\left\|\overline{\phi_{2}} \nabla_{x} \phi_{2}\right\|_{L^{2}}\left(\left\|\phi_{1}\left(\overline{\phi_{1}}-\overline{\phi_{2}}\right)\right\|_{L^{\infty}}+\left\|\overline{\phi_{2}}\left(\phi_{1}-\phi_{2}\right)\right\|_{L^{\infty}}\right) \\
\leq \frac{4 C_{1}^{3}}{\delta^{4}} M^{3}\left\|\phi_{1}-\phi_{2}\right\|_{H^{2}} .
\end{gathered}
$$

Then, taking into account (61) we just need the following bounds

$$
\begin{gathered}
\left\|\nabla_{x} \otimes \nabla_{x} \phi_{1}\left(\log \left(n_{1}\right)-\log \left(n_{2}\right)\right)\right\|_{L^{2}} \\
\leq \frac{2}{\delta}\left\|\nabla_{x} \otimes \nabla_{x} \phi_{1}\left(\phi_{1}-\phi_{2}\right)\right\|_{L^{2}} \\
\leq \frac{2 C_{1}}{\delta} M\left\|\phi_{1}-\phi_{2}\right\|_{H^{2}}, \\
\left\|\phi_{1}\left(\frac{\nabla_{x} \otimes \nabla_{x} n_{1}}{n_{1}}-\frac{\nabla_{x} n_{1} \otimes \nabla_{x} n_{1}}{n_{1}^{2}}-\frac{\nabla_{x} \otimes \nabla_{x} n_{2}}{n_{2}}+\frac{\nabla_{x} n_{2} \otimes \nabla_{x} n_{2}}{n_{2}^{2}}\right)\right\|_{L^{2}} \\
\leq \frac{2 C_{1}^{2}}{\delta^{2}} M^{2}\left(1+\frac{2 C_{1} C_{2}^{2}}{\delta^{2}} M\right)\left\|\phi_{1}-\phi_{2}\right\|_{H^{2}},
\end{gathered}
$$

$$
\begin{gathered}
\left\|\nabla_{x} \phi_{1} \otimes\left(\frac{\nabla_{x} n_{1}}{n_{1}}-\frac{\nabla_{x} n_{2}}{n_{2}}\right)\right\|_{L^{2}} \leq C_{2}^{2} M\left\|\frac{\nabla_{x} n_{1}}{n_{1}}-\frac{\nabla_{x} n_{2}}{n_{2}}\right\|_{H^{1}} \\
\leq \frac{2 C_{1} C_{2}^{2}}{\delta^{2}} M^{2}\left\{\frac{2 C_{1}}{\delta^{2}} M\left(C_{2}^{2}+C_{1} M\right)+3\right\}\left\|\phi_{1}-\phi_{2}\right\|_{H^{2}},
\end{gathered}
$$

which leads to

$$
\left\|\nabla_{x} \otimes \nabla_{x}\left[\phi_{1}\left(\log \left(n_{1}\right)-\log \left(n_{2}\right)\right)\right]\right\|_{L^{2}} \leq C(\delta, M, \Omega)\left\|\phi_{1}-\phi_{2}\right\|_{H^{2}} .
$$

We now estimate $\left\|\log \left(n_{2}\right)\left(\phi_{1}-\phi_{2}\right)\right\|_{H^{2}}$. The $L^{2}$ norm is controlled as in (64). We also have

$$
\begin{aligned}
\left\|\frac{\nabla_{x} n_{2}}{n_{2}}\left(\phi_{1}-\phi_{2}\right)\right\|_{L^{2}} & \leq \frac{C_{2}^{2}}{\delta^{2}}\left\|n_{2}\right\|_{H^{2}}\left\|\phi_{1}-\phi_{2}\right\|_{H^{2}}, \\
\left\|\log \left(n_{2}\right) \nabla_{x}\left(\phi_{1}-\phi_{2}\right)\right\|_{L^{2}} & \leq M_{1}\left\|\phi_{1}-\phi_{2}\right\|_{H^{2}} .
\end{aligned}
$$


Then, $\left\|\nabla_{x}\left[\log \left(n_{2}\right)\left(\phi_{1}-\phi_{2}\right)\right]\right\|_{L^{2}} \leq C(\delta, M, \Omega)\left\|\phi_{1}-\phi_{2}\right\|_{H^{2}}$. According to (61), there only remains to estimate

$$
\begin{gathered}
\left\|\left(\frac{\nabla_{x} \otimes \nabla_{x} n_{2}}{n_{2}}-\frac{\nabla_{x} n_{2}}{n_{2}} \otimes \frac{\nabla_{x} n_{2}}{n_{2}}\right)\left(\phi_{1}-\phi_{2}\right)\right\|_{L^{2}} \\
\leq \frac{C_{1}}{\delta^{2}}\left\|n_{2}\right\|_{H^{2}}\left(1+\frac{C_{2}^{2}}{\delta^{2}}\left\|n_{2}\right\|_{H^{2}}\right)\left\|\phi_{1}-\phi_{2}\right\|_{H^{2}}, \\
\left\|\frac{\nabla_{x} n_{2}}{n_{2}} \otimes \nabla_{x}\left(\phi_{1}-\phi_{2}\right)\right\|_{L^{2}} \leq \frac{C_{2}^{2}}{\delta^{2}}\left\|n_{2}\right\|_{H^{2}}\left\|\phi_{1}-\phi_{2}\right\|_{H^{2}}, \\
\left\|\log \left(n_{2}\right) \nabla_{x} \otimes \nabla_{x}\left(\phi_{1}-\phi_{2}\right)\right\|_{L^{2}} \leq M_{1}\left\|\phi_{1}-\phi_{2}\right\|_{H^{2}} .
\end{gathered}
$$

We then have $\| \nabla_{x} \otimes \nabla_{x}\left[\log \left(n_{2}\right)\left(\phi_{1}-\phi_{2}\right)\left\|_{L^{2}} \leq C(\delta, M, \Omega)\right\| \phi_{1}-\phi_{2} \|_{H^{2}}\right.$, for which the estimate (63) has been employed again. Now we are done with the proof.

Proof of Theorem 5.1 In order to use the Banach fixed point theorem, we shall show that the operator $\Gamma$ defined in (57) is contractive in

$$
Y_{r, T}=\left\{\phi \in B_{r, T}: \log (n) \phi-G_{B} \in C([0, T) ; D)\right\},
$$

for $r<r_{0}$ and $T<\min \left\{T_{0}, T_{1}, T_{2}\right\}$, with $r_{0}, T_{0}$ as in Lemma 5.1 and

$$
T_{1}=\frac{\alpha r}{\Lambda\left(K_{1}+\left\|G_{B}\right\|_{H^{2}}\right)}, \quad T_{2}=\frac{\alpha}{2 \Lambda K_{3}},
$$

$K_{1}, K_{3}$ chosen as in Lemma 5.2 (i) and (iii), respectively. Here, $D$ denotes the domain of the evolution semigroup defined in (56).

Step 1: $Y_{r, T}$ equipped with the distance associated with $\|\cdot\|_{L^{\infty}\left([0, T) ; H^{2}(\Omega)\right)}$ is a complete metric space. We just need to observe that $Y_{r, T}$ is closed in $B_{r, T}$. To this aim consider $\mathcal{G}$ defined in $B_{r, T}$ by

$$
\mathcal{G}[\phi](t)=\log (n(t)) \phi(t)-G_{B} \quad \forall t \in[0, T) .
$$

Since $r<r_{0}$ and $T<T_{0}$, Lemma 5.1 guarantees that $B_{r, T} \subset C\left([0, T) ; H_{\delta}^{2}\right)$. In particular, $\|\phi(t)\|_{H^{2}} \leq r_{0}+2 K_{0}$ for all $t \in[0, T)$ and applying Lemma 5.2 we find that $\mathcal{G}(t) \in H^{2}(\Omega)$ and

$$
\left\|\mathcal{G}\left[\phi_{1}\right](t)-\mathcal{G}\left[\phi_{2}\right](t)\right\|_{H^{2}} \leq K_{3}\left\|\phi_{1}(t)-\phi_{2}(t)\right\|_{H^{2}} \quad \forall t \in[0, T), \phi_{1,2} \in B_{r, T},
$$

thus $\mathcal{G}: B_{r, T} \rightarrow C\left([0, T) ; H^{2}(\Omega)\right)$ is (Lipschitz) continuous. Finally, as $C([0, T) ; D)$ is closed in $C\left([0, T) ; H^{2}(\Omega)\right)$ it is enough to notice that $Y_{r, T}=\mathcal{G}^{-1}(C([0, T) ; D))$.

Step 2: $\Gamma\left(Y_{r, T}\right) \subseteq Y_{r, T}$. Let $\phi \in Y_{r, T}$. Clearly $\Gamma(\phi)(t)=U(t)+V(t)+W_{\phi}(t) \in$ $C\left([0, T) ; H^{2}(\Omega)\right)$, where $W_{\phi} \in C([0, T) ; D)$ is given by

$$
W_{\phi}(t)=-\frac{i \Lambda}{\alpha} \int_{0}^{t} e^{A(t-s)}\left(\log (n(s)) \phi(s)-G_{B}\right) d s .
$$


Therefore

$$
\begin{aligned}
\|\Gamma(\phi)(t)-U(t)-V(t)\|_{H^{2}} & \leq \frac{\Lambda}{\alpha} \int_{0}^{t}\left\|\log (n(s)) \phi(s)-G_{B}\right\|_{H^{2}} d s \\
& \leq \frac{\Lambda}{\alpha} \int_{0}^{t}\left(K_{1}+\left\|G_{B}\right\|_{H^{2}}\right) d s \\
& \leq \frac{\Lambda}{\alpha}\left(K_{1}+\left\|G_{B}\right\|_{H^{2}}\right) T_{1}=r
\end{aligned}
$$

for all $t \in[0, T)$, thus $\Gamma(\phi) \in B_{r, T}$. Finally, in order to prove that $\Gamma(\phi) \in Y_{r, T}$ we observe that $U(t)=\overline{\phi_{B}}+e^{A t}\left(\phi_{0}-\overline{\phi_{B}}\right)$, where $\overline{\phi_{B}}$ is the harmonic extension of $\phi_{B}$ to $\Omega$, and write $\Gamma(\phi)=\overline{\phi_{B}}+\widetilde{\Gamma}(\phi)$, with

$$
\widetilde{\Gamma}(\phi)(t)=e^{A t}\left(\phi_{0}-\overline{\phi_{B}}\right)+V(t)+W_{\phi}(t) \in C([0, T) ; D) .
$$

As consequence $\Gamma(\phi)(t)_{\mid \partial \Omega} \equiv \phi_{B} \forall t \in[0, T)$ a.e. in $\partial \Omega$, which implies (along with Lemma 5.2) that $\log \left(n_{\Gamma(\phi)}\right) \Gamma(\phi)-G_{B} \in C([0, T) ; D)$. This concludes this step of the proof.

Step 3: $\Gamma: Y_{r, T} \rightarrow Y_{r, T}$ is contractive. Let $\phi_{1}, \phi_{2} \in Y_{r, T}$ and $t \in[0, T)$. Then, the isometric character of $e^{A t}: D \rightarrow D$ leads us to the estimate

$$
\left\|\Gamma\left(\phi_{1}\right)(t)-\Gamma\left(\phi_{2}\right)(t)\right\|_{H^{2}} \leq \frac{\Lambda}{\alpha} \int_{0}^{t}\left\|\left(\log \left(n_{2}(s)\right) \phi_{2}(s)-\log \left(n_{1}(s)\right) \phi_{1}(s)\right)\right\|_{H^{2}} d s .
$$

Now, according to Lemmata 5.2 (iii) and 5.1 we find

$$
\begin{aligned}
\left\|\Gamma\left(\phi_{1}\right)(t)-\Gamma\left(\phi_{2}\right)(t)\right\|_{H^{2}} & \leq \frac{\Lambda K_{3}}{\alpha} \int_{0}^{t}\left\|\phi_{1}(s)-\phi_{2}(s)\right\|_{H^{2}} d s \\
& \leq \frac{\Lambda K_{3} T_{2}}{\alpha}\left\|\phi_{1}-\phi_{2}\right\|_{L^{\infty}\left([0, T) ; H^{2}(\Omega)\right)} \\
& =\frac{1}{2}\left\|\phi_{1}-\phi_{2}\right\|_{L^{\infty}\left([0, T) ; H^{2}(\Omega)\right)},
\end{aligned}
$$

and we are done with this step of the proof.

Now, an straightforward application of Banach's theorem leads us to establish the existence of a mild solution $\phi \in Y_{r, T}$ to Eq. (53)-(54), hence $\phi(t)=U(t)+$ $V(t)+W_{\phi}(t)$ with $U, V \in X^{T}$. In particular, $U, V:[0, T) \rightarrow L^{2}(\Omega)$ are Lipschitz continuous. Besides, given $0 \leq t_{1}<t_{2}<T$ we have

$$
\begin{aligned}
\| W_{\phi}\left(t_{2}\right)- & W_{\phi}\left(t_{1}\right) \|_{L^{2}} \\
\leq & \frac{\Lambda}{\alpha}\left\{\int_{0}^{t_{1}}\left\|e^{A\left(t_{1}-s\right)}\left(I-e^{A\left(t_{2}-t_{1}\right)}\right)\left[\log (n(s)) \phi(s)-G_{B}\right]\right\|_{L^{2}} d s\right. \\
& \left.+\int_{t_{1}}^{t_{2}}\left\|e^{A\left(t_{2}-s\right)}\left[\log (n(s)) \phi(s)-G_{B}\right]\right\|_{L^{2}} d s\right\} \\
= & \frac{\Lambda}{\alpha}\left\{\int_{0}^{t_{1}}\left\|\left(I-e^{A\left(t_{2}-t_{1}\right)}\right)\left[\log (n(s)) \phi(s)-G_{B}\right]\right\|_{L^{2}} d s\right.
\end{aligned}
$$




$$
\begin{aligned}
& \left.+\int_{t_{1}}^{t_{2}}\left\|\log (n(s)) \phi(s)-G_{B}\right\|_{L^{2}} d s\right\} \\
\leq & \frac{\Lambda}{\alpha}\left\{\int_{0}^{t_{1}}\left\|\int_{0}^{t_{2}-t_{1}} e^{A \tau} \Delta_{x}[\log (n(s)) \phi(s)] d \tau\right\|_{L^{2}} d s\right. \\
& \left.+\left(K_{1}+\left\|G_{B}\right\|_{H^{2}}\right)\left(t_{2}-t_{1}\right)\right\} \\
\leq & \frac{\Lambda}{\alpha}\left(K_{1}\left(T_{0}+1\right)+\left\|G_{B}\right\|_{H^{2}}\right)\left(t_{2}-t_{1}\right),
\end{aligned}
$$

where we have used some standard properties of strongly continuous semigroups of operators (see for example [39]). The arbitrariness of $t_{1}, t_{2}$ allows to conclude that $W_{\phi}:[0, T) \rightarrow L^{2}(\Omega)$ is Lipschitz continuous, and so $\phi$. Finally, using the estimates in Lemma 5.2 (ii) we also guess that $[0, T) \ni t \rightarrow \phi(t) \log \left(n_{\phi}(t)\right)-G_{B} \in$ $L^{2}(\Omega)$ is Lipschitz continuous. Since $W_{\phi}$ is a mild solution of

$$
\begin{aligned}
& i \partial_{t} W_{\phi}=-D_{q q} \Delta_{x} W_{\phi}+\frac{\Lambda}{\alpha}\left(\log (n) \phi-G_{B}\right) \text { in }[0, T) \times \Omega, \\
& W_{\phi}(0, x)=0 \text { in } \Omega, \\
& W_{\phi}(t, x)=0 \text { in } \partial \Omega, \quad t \in[0, T),
\end{aligned}
$$

we have that $W_{\phi} \in C([0, T) ; D) \cap C^{1}\left([0, T) ; L^{2}(\Omega)\right)$, thus it is actually a strong solution. As consequence $\phi \in X^{T}$ and, since $\phi \in B_{r, T}$, then $\phi \in X_{\delta}^{T}$ in virtue of Lemma 5.1 Moreover, $\phi$ is clearly a strong solution of Eq. (53) that satisfies (54). This concludes the proof.

\section{LOCAL WELlPosedness OF THE FUll LOGARITHMiC \\ SCHRÖDINGER EquATION: END OF PROOF OF THEOREM 1.1}

We can now finish the proof of Theorem 1.1 Let $\phi_{0}=F\left(\psi_{0}\right)$ and $\phi_{B}=F\left(\psi_{B}\right)$. Using Proposition 4.1 we find that $\phi_{0} \in H_{\delta}^{2}$ and $\phi_{B} \in H_{\delta}^{3 / 2}(\partial \Omega)$. Then, Theorem 5.1 applies to yield the existence of a strong solution $\phi \in X_{\delta}^{T}$ of the initialboundary value problem associated with Eq. (53). Applying now Theorem 4.1 we can deduce that $\psi=F^{-1}(\phi) \in X_{\delta}^{T}$ is the unique solution to the corresponding problem for Eq. (3) in $[0, T)$. This proves (i). Assertion (ii) has been proved in $\S 4$. This concludes the proof of Theorem 1.1

Remark 6.1. Theorem 4.1 also applies to any equation of the Doebner-Goldin family satisfying the linearization condition (2) and to a general gauge transformation

$$
F(\phi)(t, x)=\sqrt{n_{\psi}(t, x)} \exp \left\{i\left[A(t) \log (n(t, x))+B(t) S_{\psi}(t, x)\right]\right\}
$$

that might be proved to be an homeomorphism in a quotient space of admissible states, $A(t)$ and $B(t)$ being arbitrary time-dependent functions. In this theoretical framework, the assumption that $\Omega$ be simply-connected can be removed. 
Remark 6.2. The analysis of global existence of solutions to Eq. (3) in full generality seems to be a hard task. Indeed, the eventual presence of vacuum regions $(n=0)$, which in our case are locally circumvented thanks to (H3) and the boundednes of $\Omega$, make most of our nonlinearities singular and leads to the possible formation of vortex structures.

Remark 6.3. The same difficulty arises when studying the initial value problem associated with Eq. (3)-(5) in whole space. In [42], local wellposedness was achieved for a Doebner-Goldin model on a dense subspace of $L^{2}$ under some conditions on the coefficients that make solutions to behave in a parabolic way. However, these conditions are not compatible with the linearization relations given in (2), although the techniques there developed can be adapted to our case. We postpone this research to future work.

\section{ACKNOWLEDGMENTS}

This work has been partially supported by Ministerio de Ciencia e Innovación (Spain), Project MTM2008-05271, and Junta de Andalucía, Project FQM-316.

\section{REFERENCES}

[1] Amrouche, C., Ciarlet, P. G., Ciarlet, P. Jr.: Vector and scalar potentials, Poincaré's theorem and Korn's inequality. C. R. Acad. Sci. Paris, Ser. I 345, 603-608 (2007).

[2] Amrouche, C., Girault, V.: Decomposition of vector spaces and application to the Stokes problem in arbitrary dimension. Chekoslov. Math. J. 44, 109-140 (1994).

[3] Antonelli, P., Marcati, P.: On the finite energy weak solutions to a system in Quantum Fluid Dynamics. Comm. Math. Phys. 287, 657-686 (2009).

[4] Arnold, A., Dhamo, E., Mancini, C.: The Wigner-Poisson-Fokker-Planck system: globalin-time solutions and dispersive effects. Annales de l'IHP (C)-Analyse non lineaire 24, 645-676 (2007).

[5] Arnold, A., López, J. L., Markowich, P. A., Soler, J.: An analysis of quantum FokkerPlanck models: a Wigner function approach. Rev. Mat. Iberoamericana 20, 771-814 (2004).

[6] Auberson, G., Sabatier, P. C.: On a class of homogeneous nonlinear Schrödinger equations. J. Math. Phys. 35, 4028-4040 (1994).

[7] Babin, A., Figotin, A.: Some mathematical problems in a neoclassical theory of electric charges. Discrete Cont. Dynam. Systems A 27, 1283-1326 (2010).

[8] Bacciagaluppi, G.: Nelsonian mechanics revisited. Found. Phys. Lett. 12, 1-16 (1999).

[9] Bialynicki-Birula, I., Mycielski, J.: Nonlinear wave mechanics. Ann. Phys. 100, 62-93 (1976).

[10] Brezis, H.: Analyse fonctionnelle. Masson, Paris (1983).

[11] Cañizo, J. A., López, J. L., Nieto, J.: Global $L^{1}$ theory and regularity of the 3D nonlinear Wigner-Poisson-Fokker-Planck system. J. Diff. Equ. 198, 356-373 (2004).

[12] Cazenave, T.: An introduction to nonlinear Schrödinger equations. Textos de Métodos Matemáticos 22, Rio de Janeiro (1989).

[13] Cazenave, T.: Stable solutions of the logarithmic Schrödinger equation. Nonlinear Analysis T. M. A. 7, 1127-1140 (1983).

[14] Cazenave, T., Haraux, A.: Equations d'évolution avec non linéarité logarithmique. Annals Fac. Sci. Univ. Toulouse 2, 21-55 (1980). 
[15] Cid, C., Dolbeault, J.: Defocusing nonlinear Schrödinger equation: confinement, stability and asymptotic stability. Technical report (2001).

[16] Colliander, J. , Keel, M., Staffilani, G., Takaoka, H., Tao, T.: Global well-posedness for Schrödinger equations with derivative. SIAM J. Math. Anal. 33, 649-669 (2001).

[17] Cufaro Petroni, N., De Martino, S., De Siena, S., Illuminati, F.: Stochastic-hydrodynamic model of halo formation in charged particle beams. Phys. Rev. ST Accel. Beams 6, 034206 (2003).

[18] Davidson, M. P.: A model for the stochastic origins of Schrödinger's equation. J. Math. Phys. 20, 1865-1869 (1979).

[19] Davidson, M. P.: Comments on the nonlinear Schrödinger equation. Il Nuovo Cimento B V116B, 1291-1296 (2001).

[20] De Martino, S., Falanga, M., Godano, C., Lauro, G.: Logarithmic Schrödinger-like equation as a model for magma transport. Europhys. Lett. 63, 472-475 (2003).

[21] De Martino, S., Lauro, G.: Soliton-like solutions for a capillary fluid. In: Proceedings of the 12th Conference on WASCOM, 2003, 148-152.

[22] Doebner, H. D., Goldin, G. A.: On a general nonlinear Schrödinger equation admitting diffusion currents. Phys. Lett. A 162, 397-401 (1992).

[23] Doebner, H. D., Goldin, G. A., Nattermann, P.: A family of nonlinear Schrödinger equations: linearizing transformations and resulting structure. In: Antoine, J.-P. et al. (ed.) Quantization, Coherent States and Complex Structures, 27-31. Plenum (1996).

[24] Fényes, I.: Eine wahrscheinlichkeitstheoretische begrundung und interpretation der Quantenmechanik. Z. Phys. 132, 81-103 (1952).

[25] Garbaczewski, P.: Modular Schrödinger equation and dynamical duality. Phys. Rev. E 78, 031101 (2008).

[26] Guerra, F.: Structural aspects of stochastic mechanics and stochastic field theory. Phys. Rep. 77, 263-312 (1981).

[27] Guerra, F., Pusterla, M.: A nonlinear Schrödinger equation and its relativistic generalization from basic principles. Lett. Nuovo Cimento 34, 351-356 (1982).

[28] Guerrero, P., López , J. L., Montejo-Gámez, J., Nieto, J.: A wavefunction description of stochastic-mechanical Fokker-Planck dissipation: derivation, stationary dynamics, and numerical approximation. Preprint (2011).

[29] Guerrero, P., López, J. L., Nieto, J.: Global $H^{1}$ solvability of the 3D logarithmic Schrödinger equation. Nonlinear Analysis: Real World Applications 11, 79-87 (2010).

[30] Jüngel, A., Mariani, M. C., Rial, D.: Local existence of solutions to the transient quantum hydrodynamic equations. Math. Models Meth. Appl. Sci. 12, 485-495 (2002).

[31] Kostin, M. D.: On the Schrödinger-Langevin equation. J. Chem. Phys. 57, 3589-3591 (1972); Friction and dissipative phenomena in quantum mechanics. J. Stat. Phys. 12, 145-151(1975).

[32] Lauro, G.: A note on a Korteweg fluid and the hydrodynamic form of the logarithmic Schrödinger equation. Geophys. and Astrophys. Fluid Dynamics 102, 373-380 (2008).

[33] Lindblad, G.: On the generators of quantum dynamical semigroups. Comm. Math. Phys. 48, 119-130 (1976).

[34] López, J. L.: Nonlinear Ginzburg-Landau-type approach to quantum dissipation. Phys. Rev. E. 69, 026110 (2004).

[35] López, J. L., Montejo-Gámez, J.: A hydrodynamic approach to multidimensional dissipation-based Schrödinger models from quantum Fokker-Planck dynamics, Physica D 238, 622-644 (2009).

[36] Nattermann, P., Scherer, W.: Nonlinear gauge transformations and exact solutions of the Doebner-Goldin equation. In: Doebner, H. D. et al. (ed.) Nonlinear, Deformed and Irreversible Quantum Systems, 188-199. World Scientific (1995). 
[37] Nelson, E.: Derivation of the Schrödinger equation from Newtonian Mechanics. Phys. Rev. 150, 1079-1085 (1966).

[38] Ozawa, T.: On the nonlinear Schrödinger equations of derivative type. Indiana Univ. Math. J. 45, 137-163 (1996).

[39] Pazy, A.: Semigroups of linear operators and applications to partial differential equations. Applied Mathematical Sciences 44, Springer, New York (1983).

[40] Rudin, W.: Real and complex analysis. McGraw-Hill, New York (1966).

[41] Sanin, A. L., Smirnovsky, A. A: Oscillatory motion in confined potential systems with dissipation in the context of the Schrödinger-Langevin-Kostin equation, Phys. Lett. A 372, 21-27 (2007).

[42] Teismann, H.: Square-integrable solutions to a family of nonlinear Schrödinger equations from nonlinear quantum theory. Reports Math. Phys. 56, 291-310 (2005).

[43] Wallstrom, T. C.: Inequivalence between the Schrödinger equation and the Madelung hydrodynamic equations. Phys. Rev. A 49, 1613-1617 (1994).

P. Guerrero

Centre Recerca Matemática

Campus de Bellaterra. Edifici C

08193 Bellaterra (BARCELONA)

SPAIN

E-mail address: pguerrero@crm.cat

J. L. LÓPEZ

Departamento de Matemática Aplicada

Facultad DE Ciencias

UNIVERSIDAD DE GRANADA

18071 GRANADA

SPAIN

E-mail address: jllopez@ugr.es

J. Montejo-Gámez

Departamento de Matemática Aplicada

Facultad de Ciencias

UNIVERSIDAD DE GRANADA

18071 Granada

SPAIN

E-mail address: jmontejo@ugr.es

J. NiETO

Departamento de Matemática Aplicada

18071 GRANADA

SPAIN

E-mail address: jmnieto@ugr.es 